This is a postprint version of the following published document:

Koch, Tobias; Vázquez-Vilar, Gonzalo. A Rigorous Approach to HighResolution Entropy-Constrained Vector Quantization, in: IEEE Transactions on Information Theory, 64(4), April 2018, pp. 2609-2625

DOI: https://doi.org/10.1109/TIT.2018.2803064

C2018 IEEE. Personal use of this material is permitted. Permission from IEEE must be obtained for all other uses, in any current or future media, including reprinting/republishing this material for advertising or promotional purposes, creating new collective works, for resale or redistribution to servers or lists, or reuse of any copyrighted component of this work in other works. 


\title{
A Rigorous Approach to High-Resolution Entropy-Constrained Vector Quantization
}

\author{
Tobias Koch, Senior Member, IEEE, and Gonzalo Vazquez-Vilar, Member, IEEE
}

\begin{abstract}
The nonnegativity of relative entropy implies that the differential entropy of a random vector $X$ with probability density function (pdf) $f$ is upper-bounded by $-E[\log g(\mathbf{X})]$ for any arbitrary pdf $g$. Using this inequality with a cleverly chosen $g$, we derive a lower bound on the asymptotic excess rate of entropy-constrained vector quantization for $d$-dimensional sources and $r$ th-power distortion, where the asymptotic excess rate is defined as the difference between the smallest output entropy of a vector quantizer satisfying the distortion constraint and the rate-distortion function in the limit as the distortion tends to zero. Specialized to the one-dimensional case, this lower bound coincides with the asymptotic excess rate achieved by a uniform quantizer, thereby recovering the result by Gish and Pierce that uniform quantizers are asymptotically optimal as the allowed distortion tends to zero. Furthermore, in the one-dimensional case the derivation of the lower bound reveals a necessary condition for a sequence of quantizers to be asymptotically optimal. This condition implies that any sequence of asymptotically-optimal almost-regular quantizers must converge to a uniform quantizer as the distortion tends to zero. While the obtained lower bound itself is not novel, to the best of our knowledge, we present the first rigorous derivation that follows the direct approach by Gish and Pierce without resorting to heuristic high-resolution approximations commonly found in the quantization literature. Furthermore, our derivation holds for all $d$-dimensional sources having finite differential entropy and whose integer part has finite entropy. In contrast to Gish and Pierce, we do not require additional constraints on the continuity or decay of the source pdf.
\end{abstract}

Index Terms-Entropy constrained, high resolution, quantization, rate-distortion theory.

\section{INTRODUCTION}

A VECTOR quantizer for a source $\mathbf{X}$ with probability distribution $P_{\mathbf{X}}$ is a (deterministic) mapping $q(\cdot)$ from the source alphabet $\mathcal{X}$ to the (countable) reconstruction alphabet $\hat{\mathcal{X}}$. The performance of a quantizer is typically measured by the distortion and the rate. The former assesses the quantizer's

This work has received funding from the European Research Council (ERC) under the European Union's Horizon 2020 research and innovation programme (grant agreement number 714161), from the 7th European Union Framework Programme under Grant 333680, from the Ministerio de Economía y Competitividad of Spain under Grants TEC2013-41718-R, RYC-201416332, IJCI-2015-27020, TEC2015-69648-REDC, and TEC2016-78434-C33-R (AEI/FEDER, EU), and from the Comunidad de Madrid under Grant S2103/ICE-2845. The material in this paper was presented in part at the 2016 IEEE International Symposium on Information Theory, Barcelona, Spain, July 2016.

The authors are with the Signal Theory and Communications Department, Universidad Carlos III de Madrid, 28911, Leganés, Spain and also with the Gregorio Marañón Health Research Institute, 28007, Madrid, Spain (e-mails: koch@tsc.uc3m.es and gvazquez@tsc.uc3m.es).

Copyright (c) 2018 IEEE. Personal use of this material is permitted. However, permission to use this material for any other purposes must be obtained from the IEEE by sending a request to pubs-permissions @ieee.org. resolution and the latter indicates the number of bits required to describe the quantizer output. In this paper, we assume that the source and reconstruction alphabets are subsets of the $d$ dimensional Euclidean space, i.e., $\mathcal{X}, \hat{\mathcal{X}} \subseteq \mathbb{R}^{d}$. We consider the $r$ th-power distortion $\mathrm{E}\left[\|\mathbf{X}-q(\mathbf{X})\|^{r}\right]$, where $\|\cdot\|$ is an arbitrary norm on $\mathbb{R}^{d}$ and $r>0$ is an arbitrary exponent, and we define the rate of the quantizer as the entropy of its output $H(q(\mathbf{X}))$, which by Shannon's source coding theorem [1] is roughly the average length of the shortest variablelength source code that describes the quantizer output. ${ }^{1} \mathrm{~A}$ quantizer whose rate is measured by the entropy of its output is sometimes referred to as an entropy-constrained quantizer.

To describe the tradeoff between distortion and rate, we define the smallest rate of an entropy-constrained quantizer satisfying the distortion constraint $D$ by

$$
R_{r, d}(D) \triangleq \inf _{q(\cdot)} H(q(\mathbf{X}))
$$

where the infimum is over the set of quantizers $q(\cdot)$ satisfying

$$
\mathrm{E}\left[\|\mathbf{X}-q(\mathbf{X})\|^{r}\right] \leq D
$$

We shall benchmark the rate $R_{r, d}(D)$ of the entropyconstrained quantizer against the rate-distortion function ${ }^{2}$ $R(D)$, defined as

$$
R(D) \triangleq \inf _{P_{\hat{\mathbf{X}} \mid \mathbf{X}}} I(\mathbf{X} ; \hat{\mathbf{X}})
$$

where the infimum is over all conditional distributions of $\hat{\mathbf{X}}$ given $\mathbf{X}$ for which

$$
\mathrm{E}\left[\|\mathbf{X}-\hat{\mathbf{X}}\|^{r}\right] \leq D
$$

Note that $R_{r, d}(D) \geq R(D)$. Indeed, $\mathbf{X}$ determines the quantizer output $q(\mathbf{X})$, so we have $H(q(\mathbf{X}) \mid \mathbf{X})=0$ and (1) can be written as

$$
R_{r, d}(D)=\inf _{q(\cdot)} I(\mathbf{X} ; q(\mathbf{X})) .
$$

Since $\hat{\mathbf{X}}=q(\mathbf{X})$ corresponds to a deterministic $P_{\hat{\mathbf{X}} \mid \mathbf{X}}$, the claim follows by comparing (3) with (5). To simplify notation, we shall sometimes substitute $\hat{\mathbf{X}}=q(\mathbf{X})$ in our analysis of $R_{r, d}(D)$.

\footnotetext{
${ }^{1}$ The shortest uniquely decodable source code describing $q(\mathbf{X})$ has an average length that is between $H(q(\mathbf{X}))$ and $H(q(\mathbf{X}))+1$, where a source code is said to be uniquely decodable if any concatenation of codewords has only one possible string of source symbols producing it [2, Chapter 5]. If the assumption of unique decodability is dropped, then the shortest source code has an average length that is between $H(q(\mathbf{X}))-\log (1+H(q(\mathbf{X})))-\log e$ and $H(q(\mathbf{X}))$ [3], [4].

${ }^{2}$ In the literature, $R(D)$ is sometimes also referred to as the Shannon ratedistortion function or the information rate-distortion function.
} 
Intuitively, the rate-distortion function $R(D)$ describes the smallest rate of a vector quantizer that jointly quantizes a block of $n$ source symbols with an expected $r$ th-power distortion no larger than $D$. More precisely, suppose a source produces the sequence of independent and identically distributed source symbols $\left\{\mathbf{X}_{k}, k \in \mathbb{Z}\right\}$ according to a distribution $P_{\mathbf{X}}$ and, for some blocklength $n$, we employ a vector quantizer that jointly quantizes a block of $n$ source symbols $\mathbf{X}_{1}, \ldots, \mathbf{X}_{n}$ to one of $e^{n R}$ possible sequences of quantized symbols $\hat{\mathbf{X}}_{1}, \ldots, \hat{\mathbf{X}}_{n}$. The rate-distortion function $R(D)$ is the smallest rate $R$ for which there exists a blocklength $n$ and a vector quantizer satisfying [5]

$$
\frac{1}{n} \sum_{k=1}^{n} \mathrm{E}\left[\left\|\mathbf{X}_{k}-\hat{\mathbf{X}}_{k}\right\|^{r}\right] \leq D .
$$

In this paper, we consider the quantization of a $d$ dimensional, real-valued source vector $\mathbf{X}$ with probability density function (pdf) $f_{\mathbf{X}}$ and focus on the asymptotic ratedistortion tradeoff in the limit as the permitted distortion tends to zero. Specifically, we study the asymptotic excess rate with respect to the rate-distortion function, defined as

$$
\mathrm{R}_{r, d} \triangleq \underline{\lim _{D \downarrow 0}}\left\{R_{r, d}(D)-R(D)\right\}
$$

where lim denotes the limit inferior. The considered setup is sufficiently general to comprise various problems of interest in high-resolution vector quantization. For example, it allows us to analyze the performance of quantization schemes that buffer $d$ consecutive symbols of a one-dimensional memoryless source and then quantize them using a $d$-dimensional vector quantizer.

For one-dimensional sources $(d=1)$ and squared-error distortion $(r=2$ and $\|a\|=|a|, a \in \mathbb{R})$, Gish and Pierce demonstrated that the asymptotic excess rate is equal to [6]

$$
\mathrm{R}_{2,1}=\frac{1}{2} \log \frac{\pi e}{6}
$$

where $\log (\cdot)$ denotes the natural logarithm. In fact, (8) holds for any arbitrary norm since all norms on $\mathbb{R}$ are proportional to the absolute value. They further showed that this excess rate can be achieved by a uniform quantizer, hence the well-known result that "uniform quantizers are asymptotically optimal as the allowed distortion tends to zero."3 For multi-dimensional sources, only bounds on $\mathrm{R}_{r, d}$ are available. In particular, a lower bound on $\mathrm{R}_{r, d}$ for general $r$ and $d$ was presented by Yamada et al. [8, eq. (55)]

$$
\mathrm{R}_{r, d} \geq \frac{d}{r} \log \left(\frac{\Gamma(1+d / r)^{r / d} e}{1+d / r}\right)
$$

where

$$
\Gamma(\alpha) \triangleq \int_{0}^{\infty} e^{-t} t^{\alpha-1} \mathrm{~d} t, \quad \alpha>0
$$

denotes the Gamma function. For one-dimensional sources and quadratic distortion $(r=2)$, the lower bound (9) coincides with the excess rate (8) obtained by Gish and Pierce.

\footnotetext{
${ }^{3}$ The fact that, in the high-resolution case, the expected quadratic distortion of uniform scalar quantization exceeds the least distortion achievable by any quantization scheme by a factor of only $\pi e / 6$ was already discovered by Koshelev in 1963. See [7] and references therein for more details.
}

To prove (8), Gish and Pierce [6] impose constraints on the continuity and decay of the pdf of X. Furthermore, they merely provide an intuitive explanation of their converse result together with an outline of the proof - at the end of [6, Appendix II] they write "The complete proof is surprisingly long and will not be given here." Similarly, the derivation of (9) by Yamada et al. is based on heuristic high-resolution approximations commonly found in the quantization literature.

The result (8) is equivalent to a result by Zador [9], which concerns the asymptotic excess distortion with respect to the distortion-rate function as the rate tends to infinity. Indeed, let $D_{r, d}(R)$ denote the minimum distortion achievable with a vector quantizer whose output has an entropy not exceeding $R$, i.e.,

$$
D_{r, d}(R) \triangleq \inf _{q(\cdot)} \mathrm{E}\left[\|\mathbf{X}-q(\mathbf{X})\|^{r}\right]
$$

where the infimum is over the set of quantizers $q(\cdot)$ satisfying $H(q(\mathbf{X})) \leq R$. Zador's theorem states that

$$
\lim _{R \rightarrow \infty} e^{\frac{r}{d} R} D_{r, d}(R)=b_{r, d} e^{\frac{r}{d} h(\mathbf{X})}
$$

where $b_{r, d}$ is a constant that only depends on $r$ and $d$ but not on the distribution of $\mathbf{X}$. Zador did not evaluate the constant $b_{r, d}$, but he showed that

$$
\frac{1}{(1+r / d) V_{d}^{r / d}} \leq b_{r, d} \leq \frac{\Gamma(1+r / d)}{V_{d}^{r / d}}
$$

where $V_{d}$ denotes the volume of the $d$-dimensional unit ball $\left\{\mathbf{x} \in \mathbb{R}^{d}:\|\mathbf{x}\| \leq 1\right\}$. For one-dimensional sources and squared-error distortion, it can be further shown that $b_{2,1}=1 / 12$. Taking logarithms on both sides of (12), and replacing $R \leftrightarrow R_{r, d}(D)$ and $D_{r, d}(R) \leftrightarrow D$, we thus obtain that

$$
R_{r, d}(D)=h(\mathbf{X})+\frac{d}{r} \log \frac{1}{D}+\frac{d}{r} \log b_{r, d}+o_{R}(1)
$$

where $o_{R}(1)$ denotes error terms that vanish as $R$ tends to infinity. Furthermore, the rate-distortion function $R(D)$ can be lower-bounded as [8, eq. (50)]

$$
\begin{aligned}
R(D) \geq & h(\mathbf{X})+\frac{d}{r} \log \frac{1}{D} \\
& -\frac{d}{r} \log \left(\frac{r}{d}\left(V_{d} \Gamma(1+d / r)\right)^{r / d} e\right) .
\end{aligned}
$$

The right-hand side (RHS) of (15) is referred to as Shannon lower bound. It has been demonstrated that its difference to $R(D)$ vanishes as $D$ tends to zero, provided that the source distribution satisfies certain conditions; see, e.g., [10][12]. A finite-blocklength refinement of this bound can be found in [13], [14]. Recently, it has been demonstrated that the Shannon lower bound is asymptotically tight for sources having finite differential entropy and whose integer part has finite entropy [12]. Thus, we have for the class of sources considered in this paper (which will be specified in detail in Section II)

$$
\begin{aligned}
R(D)= & h(\mathbf{X})+\frac{d}{r} \log \frac{1}{D} \\
& -\frac{d}{r} \log \left(\frac{r}{d}\left(V_{d} \Gamma(1+d / r)\right)^{r / d} e\right)+o_{D}(1)
\end{aligned}
$$


where $o_{D}(1)$ denotes error terms that vanish as $D$ tends to zero. Combining (14) with (16), we obtain

$$
\mathrm{R}_{r, d}=\frac{d}{r} \log \left(b_{r, d} \frac{r}{d}\left(V_{d} \Gamma(1+d / r)\right)^{r / d} e\right) .
$$

For one-dimensional sources and squared-error distortion, the equivalence of Zador's theorem (12) and Gish and Pierce's result (8) thus follows by noting that $b_{2,1}=1 / 12, V_{1}=2$, and $\Gamma(3 / 2)=\sqrt{\pi} / 2$. Furthermore, lower-bounding $b_{r, d}$ using the left-most inequality in (13) recovers (9).

While Zador's original proof of (12) was flawed, a rigorous proof was given by Gray et al. [15] by using a Langrangian formulation of variable-rate vector quantization. Their proof follows Zador's approach of 1) proving the result for sources with a uniform pdf on the unit cube; 2) extending it to piecewise constant pdfs on disjoint cubes of equal sides; 3 ) proving the result for a general pdf on a cube; and 4) proving the result for general pdfs. Gray et al. do not impose any constraints on the continuity or decay of the pdf of $\mathbf{X}$, so their proof is more general than the proofs by Zador [9] and by Gish and Pierce [6].

In this paper, we derive the lower bound (9) on $\mathrm{R}_{r, d}$ without resorting to heuristic high-resolution approximations. In contrast to [15], our proof follows essentially along the lines outlined by Gish and Pierce [6]. We do not impose any constraints on the continuity or decay of the pdf of $\mathbf{X}$, so our proof holds under the same conditions on the source as the proof by Gray et al., and it is more general than the proof by Gish and Pierce.

For one-dimensional sources, the derivation of the lower bound reveals a necessary condition for a sequence of quantizers (parametrized by $D$ ) to achieve the asymptotic excess rate $\mathrm{R}_{r, 1}$. We apply this condition to the family of almost-regular quantizers, which was introduced by György and Linder in [16] and includes the uniform quantizers. Almost-regular quantizers are relevant because they achieve $D_{r, 1}(R)$ when $r \geq 1$ [16, Theorem 3]. Thus, for one-dimensional sources and $r$ th-power distorion with $r \geq 1$, we can restrict ourselves to almost-regular quantizers without loss of optimality. The necessary condition implies that any sequence of almostregular quantizers achieving $\mathrm{R}_{r, 1}$ must converge to a uniform quantizer as $D \rightarrow 0$. This suggests that asymptotically-optimal quantizers must essentially be uniform.

The rest of this paper is organized as follows. Section II introduces the problem setup and presents the main result of this paper, Theorem 1. Section III provides a back-of-theenvelope derivation of Theorem 1 that serves as an outline for the proof. Section IV contains the complete proof of this theorem. Section $\mathrm{V}$ presents a necessary condition for a sequence of quantizers to achieve the asymptotic excess rate. Section VI assesses the tightness of the lower bound presented in Theorem 1 for multi-dimensional sources by numerically comparing it to several upper bounds achievable by lattice quantizers. Section VII concludes the paper with a summary and discussion of the results.

\section{Problem Setup And Main Result}

We consider a $d$-dimensional, real-valued source $\mathbf{X}$ with support $\mathcal{X} \subseteq \mathbb{R}^{d}$ whose distribution is absolutely continuous with respect to the Lebesgue measure, and we denote its pdf by $f_{\mathbf{X}}$. We require the source to satisfy the following two conditions:

C1 The differential entropy

$$
h(\mathbf{X}) \triangleq-\int_{\mathcal{X}} f_{\mathbf{X}}(\mathbf{x}) \log f_{\mathbf{X}}(\mathbf{x}) \mathrm{d} \mathbf{x}
$$

is well-defined and finite;

$\mathrm{C} 2$ the integer part of the source $\mathbf{X}$ has finite entropy, i.e.,

$$
H(\lfloor\mathbf{X}\rfloor)<\infty \text {. }
$$

Here $\lfloor\mathbf{a}\rfloor, \mathbf{a}=\left(a_{1}, \ldots, a_{d}\right) \in \mathbb{R}^{d}$ denotes the elementwise floor function, i.e., $\lfloor\mathbf{a}\rfloor=\left(\left\lfloor a_{1}\right\rfloor, \ldots,\left\lfloor a_{d}\right\rfloor\right)$ where $\left\lfloor a_{\ell}\right\rfloor$ denotes the largest integer less than or equal to $a_{\ell}$.

Condition $\mathrm{C} 2$ requires that quantizing the source with a cubic lattice quantizer of unit-volume cells gives rise to a discrete random variable of finite entropy. This is necessary for the asymptotic excess rate $\mathrm{R}_{r, d}$ to be well-defined. Indeed, as demonstrated in [12], if $H(\lfloor\mathbf{X}\rfloor)=\infty$ then the rate-distortion function $R(D)$ is infinite for any $D$. Since $R_{r, d}(D) \geq R(D)$, this implies that in this case $R_{r, s}(D)-R(D)$ is of the form $\infty-\infty$. Fortunately, Condition C2 is very mild. For example, by generalizing [17, Proposition 1] to the vector case, it can be shown that it is satisfied if $E[\log (1+\|\mathbf{X}\|)]<\infty$. This in turn is true, for example, for sources for which $E\left[\|\mathbf{X}\|^{\alpha}\right]<\infty$ for some $\alpha>0$.

The quantity $H(\lfloor\mathbf{X}\rfloor)$ is intimately related with the Rényi information dimension, defined as [18] (see also [17], [19])

$$
d(\mathbf{X}) \triangleq \lim _{m \rightarrow \infty} \frac{H(\lfloor m \mathbf{X}\rfloor / m)}{\log m}
$$

if the limit exists. (If the limit does not exist, then one can define the upper and lower Rényi information dimension by replacing the limit by the limit superior and limit inferior, respectively.) Indeed, it can be shown that a source vector has finite Rényi information dimension if, and only if, (19) is satisfied [18], [17, Proposition 1].

The quantizer is characterized by the (Borel measurable) function $q: \mathcal{X} \rightarrow \hat{\mathcal{X}}$ for some countable reconstruction alphabet $\hat{\mathcal{X}} \subseteq \mathbb{R}^{d}$. Equivalently, we characterize $q(\cdot)$ by the quantization regions $\left\{\mathcal{S}_{i}\right\}$ and corresponding reconstruction values $\left\{\hat{\mathbf{x}}_{i}\right\}$, where the number of quantization regions may be infinite. Specifically, $\left\{\mathcal{S}_{i}\right\}$ are disjoint (Borel measurable) subsets of $\mathbb{R}^{d}$ that together with the reconstruction values $\left\{\hat{\mathbf{x}}_{i}\right\}$ satisfy

$$
\begin{aligned}
& \bigcup_{i} \mathcal{S}_{i}=\mathcal{X} \\
& q(\mathbf{x})=\sum_{i} \hat{\mathbf{x}}_{i} \mathbf{1}\left\{\mathbf{x} \in \mathcal{S}_{i}\right\}, \quad \text { for } \mathbf{x} \in \mathcal{X}
\end{aligned}
$$

where $\mathbf{1}\{\cdot\}$ denotes the indicator function. To simplify notation, we denote the Lebesgue measure of the quantization region $\mathcal{S}_{i}$ by $\Delta_{i}$ and the probability of $\mathbf{X}$ being in $\mathcal{S}_{i}$ by $p_{i}$.

The main result of this paper is a rigorous derivation of the lower bound (9) on the excess rate $\mathrm{R}_{r, d}$ for general $r$ and $d$. In contrast to [8], our derivation does not resort to heuristic highresolution approximations. Furthermore, in contrast to [6], our bound does not require any continuity or decay conditions on 
the behavior of the source pdf. It holds for all source vectors having a pdf, having finite differential entropy, and having finite Rényi information dimension.

Theorem 1 (Main Result): Let the source vector $\mathbf{X}$ have a pdf, and assume that $h(\mathbf{X})$ and $H(\lfloor\mathbf{X}\rfloor)$ are finite. Then, the asymptotic excess rate $\mathrm{R}_{r, d}$, as defined in (7), is lower-bounded by

$$
\mathrm{R}_{r, d} \geq \frac{d}{r} \log \left(\frac{\Gamma(1+d / r)^{r / d} e}{1+d / r}\right) .
$$

Proof: See Section IV.

In the one-dimensional case, (22) becomes

$$
\mathrm{R}_{r, 1} \geq \frac{1}{r} \log \left(\frac{\Gamma(1+1 / r)^{r} e}{1+1 / r}\right) .
$$

As we shall see next, (23) can be achieved by a uniform quantizer, so in the one-dimensional case the lower bound (22) is tight. Furthermore, for quadratic distortion, (23) is equal to $1 / 2 \log (\pi e / 6)$, hence it recovers the excess rate obtained by Gish and Pierce.

To demonstrate the tightness of (23), and to assess the accuracy of (22) in higher-dimensional cases, we consider an upper bound on the asymptotic excess rate that follows by restricting ourselves to the class of tessellating quantizers. A polytope $\mathcal{P}$ is tessellating if there exists a partition of $\mathbb{R}^{d}$ consisting of translated and/or rotated copies of $\mathcal{P}$; a tessellating quantizer, denoted by $q_{\mathcal{P}}: \mathcal{X} \rightarrow \hat{\mathcal{X}}$, is a quantizer whose quantization regions $\mathcal{S}_{i}$ are translated and/or rotated copies of a tessellating convex polytope $\mathcal{P}$ and the corresponding reconstruction values $\hat{\mathbf{x}}_{i}$ are the centroids of $\mathcal{S}_{i}$. A special case of a tessellating quantizer is a lattice quantizer, i.e., a quantizer whose quantization regions are the Voronoi cells of a $d$-dimensional lattice. Note that in the one-dimensional case the only convex polytope is the interval, so in this case the tessellating quantizer is the uniform quantizer. For the class of tessellating quantizers, Linder and Zeger [20] derived an asymptotic expression equivalent to (12).

Theorem 2 (Linder and Zeger [20, Theorem 1]): Let the source vector $\mathbf{X}$ have a pdf, and assume that $h(\mathbf{X})$ and $H(\lfloor\mathbf{X}\rfloor)$ are finite. Then, a tessellating quantizer $q_{\mathcal{P}}(\cdot)$ with $r$ th-power distortion $\mathrm{E}\left[\left\|\mathbf{X}-q_{\mathcal{P}}(\mathbf{X})\right\|^{r}\right]=D$ and rate $R_{\mathcal{P}}(D) \triangleq H\left(q_{\mathcal{P}}(\mathbf{X})\right)$ satisfies

$$
\lim _{D \downarrow 0} D e^{\frac{r}{d} R_{\mathcal{P}}(D)}=\ell(\mathcal{P}) e^{\frac{r}{d} h(\mathbf{X})}
$$

where $\ell(\mathcal{P})$ denotes the normalized $r$-th moment of $\mathcal{P}$, defined as

$$
\ell(\mathcal{P}) \triangleq \frac{\int_{\mathcal{P}}\left\|\mathbf{x}-\overline{\mathbf{x}}_{\mathcal{P}}\right\|^{r} \mathrm{~d} \mathbf{x}}{V(\mathcal{P})^{1+r / d}}
$$

with $V(\mathcal{P})$ and $\overline{\mathbf{x}}_{\mathcal{P}}$ denoting the volume and centroid of $\mathcal{P}$, respectively.

Remark: To be precise, [20, Theorem 1] requires that $H\left(q_{\mathcal{P}_{\alpha}}(\mathbf{X})\right)<\infty$ for some $\alpha>0$ rather than $H(\lfloor\mathbf{X}\rfloor)<\infty$. (Here, $\mathcal{P}_{\alpha}=\left\{x \in \mathbb{R}^{d}: x / \alpha \in \mathcal{P}\right\}$ denotes the polytope $\mathcal{P}$ rescaled by $\alpha$.) Nevertheless, its proof hinges on a lemma by Csiszár (cf. [20, Lemma 2]), which also applies if the condition $H\left(q_{\mathcal{P}_{\alpha}}(\mathbf{X})\right)<\infty$ is replaced by $H(\lfloor\mathbf{X}\rfloor)<\infty$. Specifically, by setting in [20, Lemma 2] the partition $\mathcal{B}_{0}=\left\{B_{1}, B_{2}, \ldots\right\}$ of $\mathbb{R}^{d}$ to be the set of $d$-dimensional cubes of unit-volume with the lower-most cornerpoint located at coordinates $\mathbf{i} \in \mathbb{Z}^{d}$, this partition satisfies the lemma's conditions provided that $H(\lfloor\mathbf{X}\rfloor)$ is finite.

Taking logarithms on both sides of (24), we obtain

$$
R_{\mathcal{P}}(D)=h(\mathbf{X})+\frac{d}{r} \log \frac{1}{D}+\frac{d}{r} \log \ell(\mathcal{P})+o_{D}(1) .
$$

Combining (26) with (16), we obtain

$$
\begin{aligned}
& \lim _{D \downarrow 0}\left\{R_{\mathcal{P}}(D)-R(D)\right\} \\
& \quad=\frac{d}{r} \log \left(\frac{r}{d}\left(V_{d} \Gamma(1+d / r)\right)^{r / d} e\right)+\frac{d}{r} \log \ell(\mathcal{P}) .
\end{aligned}
$$

Since a tessellating quantizer with $r$ th-power distortion $D$ satisfies (2), the rate $R_{\mathcal{P}}(D)$ upper-bounds $R_{r, d}(D)$. It follows that

$$
\mathrm{R}_{r, d} \leq \frac{d}{r} \log \left(\frac{r}{d}\left(V_{d} \Gamma(1+d / r)\right)^{r / d} e\right)+\frac{d}{r} \log \ell(\mathcal{P})
$$

for any $d$-dimensional, tessellating, convex polytope $\mathcal{P}$.

Using that in the one-dimensional case the only convex polytope is the interval, and noting that the interval has the normalized $r$-th moment

$$
\ell(\mathcal{P})=\frac{1}{V_{d}^{r}(1+r)}
$$

the upper bound (28) coincides in this case with the RHS of (23). Thus, in the one-dimensional case a tessellating quantizer (which in this case is the uniform quantizer) is asymptotically optimal.

\section{DERIVATION FOR OnE-Dimensional Sources AND CERTAIN QUANTIZERS}

Before proving Theorem 1, we provide a simplified derivation of the lower bound (22) for one-dimensional sources $(d=1)$ and squared-error distortion $(r=2$ and $\|a\|=|a|$, $a \in \mathbb{R})$ that will serve as an outline for the complete proof of Theorem 1 given in Section IV. Specialized to this setting, Theorem 1 becomes

$$
\mathrm{R}_{2,1} \geq \frac{1}{2} \log \frac{\pi e}{6}
$$

In our derivation we shall only consider quantizers that satisfy

$$
\sup _{i} \sup _{x \in \mathcal{S}_{i}}\left(x-\hat{x}_{i}\right)^{2} \leq \alpha D, \quad \text { for some constant } \alpha .
$$

This simplifying assumption is, for example, satisfied by the uniform quantizer when $\hat{x}_{i}$ is the midpoint of $\mathcal{S}_{i}$ and the cell length $\Delta$ vanishes proportionally to $\sqrt{D}$. However, it is prima facie unclear whether (31) holds without loss of optimality for general sources.

By (5), we have

$$
R_{2,1}(D)=h(X)-\sup _{q(\cdot)} h(X \mid \hat{X}) .
$$

We upper-bound $h(X \mid \hat{X})$ by using that, given $\hat{X}=\hat{x}_{i}$, the support of $X$ is $\mathcal{S}_{i}$, so a uniform distribution over $\mathcal{S}_{i}$ maximizes the differential entropy [2, Theorem 11.1.1]:

$$
h\left(X \mid \hat{X}=\hat{x}_{i}\right) \leq \log \Delta_{i} .
$$


Averaging over $\hat{X}$ then yields

$$
R_{2,1}(D) \geq h(X)-\sup _{q(\cdot)} \sum_{i} p_{i} \log \Delta_{i} .
$$

By Jensen's inequality, this can be further lower-bounded by

$$
R_{2,1}(D) \geq h(X)-\frac{1}{2} \log \left(\sup _{q(\cdot)} \sum_{i} p_{i} \Delta_{i}^{2}\right) .
$$

Together with (16) specialized to the case $d=1$ and $r=2$, this yields

$$
\begin{aligned}
& \frac{\lim _{D \downarrow 0}}{}\left\{R_{2,1}(D)-R(D)\right\} \\
& \quad \geq \frac{\lim }{D \downarrow 0}\left\{\frac{1}{2} \log (2 \pi e D)-\frac{1}{2} \log \left(\sup _{q(\cdot)} \sum_{i} p_{i} \Delta_{i}^{2}\right)\right\} .
\end{aligned}
$$

In order to prove (30), it remains to show that, for any sequence of quantizers (parametrized by $D$ ),

$$
\varlimsup_{D \downarrow 0} \frac{1}{D} \sum_{i} p_{i} \Delta_{i}^{2} \leq 12
$$

where $\overline{l i m}$ denotes the limit superior. Then the RHS of (36) is lower-bounded by $1 / 2 \log (\pi e / 6)$ and we obtain (30) upon noting that the left-hand side (LHS) of (36) is equal to $R_{2,1}$. Hence, we recover Theorem 1 for one-dimensional sources and squared-error distortion.

The upper bound (37) follows along the lines of the proof of [20, Lemma 1]. We first express $\mathrm{E}\left[(X-\hat{X})^{2}\right]$ as

$$
\begin{aligned}
\mathrm{E}\left[(X-\hat{X})^{2}\right]= & \sum_{i} \int_{\mathcal{S}_{i}} f_{X}(x)\left(x-\hat{x}_{i}\right)^{2} \mathrm{~d} x \\
= & \sum_{i} \frac{p_{i}}{\Delta_{i}} \int_{\mathcal{S}_{i}}\left(x-\hat{x}_{i}\right)^{2} \mathrm{~d} x \\
& -\sum_{i} \int_{\mathcal{S}_{i}}\left[\frac{p_{i}}{\Delta_{i}}-f_{X}(x)\right]\left(x-\hat{x}_{i}\right)^{2} \mathrm{~d} x .
\end{aligned}
$$

We next note that the region $\mathcal{S}_{i}$ of measure $\Delta_{i}$ that minimizes $\int_{\mathcal{S}_{i}}\left(x-\hat{x}_{i}\right)^{2} \mathrm{~d} x$ is the interval $\left[\hat{x}_{i}-\frac{\Delta_{i}}{2}, \hat{x}_{i}+\frac{\Delta_{i}}{2}\right]$, so

$$
\frac{1}{\Delta_{i}} \int_{\mathcal{S}_{i}}(x-\hat{x})^{2} \mathrm{~d} x \geq \frac{\Delta_{i}^{2}}{12} .
$$

The first term on the RHS of (38) can therefore be lowerbounded by

$$
\sum_{i} \frac{p_{i}}{\Delta_{i}} \int_{\mathcal{S}_{i}}\left(x-\hat{x}_{i}\right)^{2} \mathrm{~d} x \geq \sum_{i} p_{i} \frac{\Delta_{i}^{2}}{12} .
$$

To evaluate the second term on the RHS of (38), we introduce the piecewise-constant pdf

$$
f_{X}^{(\Delta)}(x) \triangleq \sum_{i} \frac{p_{i}}{\Delta_{i}} \mathbf{1}\left\{x \in \mathcal{S}_{i}\right\}, \quad x \in \mathbb{R} .
$$

With this, we can upper-bound the second term on the RHS of (38) as

$$
\begin{aligned}
& \sum_{i} \int_{\mathcal{S}_{i}}\left[\frac{p_{i}}{\Delta_{i}}-f_{X}(x)\right]\left(x-\hat{x}_{i}\right)^{2} \mathrm{~d} x \\
& \quad=\sum_{i} \int_{\mathcal{S}_{i}}\left[f_{X}^{(\Delta)}(x)-f_{X}(x)\right]\left(x-\hat{x}_{i}\right)^{2} \mathrm{~d} x \\
& \quad \leq \alpha D \int\left|f_{X}^{(\Delta)}(x)-f_{X}(x)\right| \mathrm{d} x
\end{aligned}
$$

since, by the assumption (31), we have $\left(x-\hat{x}_{i}\right)^{2} \leq \alpha D$ for every $x \in \mathcal{S}_{i}$.

By Lebesgue's differentiation theorem, $f_{X}^{(\Delta)}$ converges to $f_{X}$ almost everywhere as $\sup _{i} \Delta_{i} \rightarrow 0$. It therefore follows from Scheffe's Lemma [21, Theorem 16.12] that

$$
\lim _{D \downarrow 0} \int\left|f_{X}^{(\Delta)}(x)-f_{X}(x)\right| \mathrm{d} x=0 .
$$

Combining (40) and (42) with (38), and applying the distortion constraint $\mathrm{E}\left[(X-\hat{X})^{2}\right] \leq D$, we obtain

$$
\sum_{i} p_{i} \Delta_{i}^{2} \leq 12 D\left(1+\alpha \int\left|f_{X}^{(\Delta)}(x)-f_{X}(x)\right| \mathrm{d} x\right) .
$$

Together with (43) this gives (37).

\section{Proof of Theorem 1}

The above back-of-the-envelope derivation directly generalizes to multi-dimensional sources and $r$ th-power distortion. In order to prove Theorem 1, it would remain to show that (31) holds without loss of optimality. Unfortunately, for general sources this appears to be a difficult task. Indeed, the quantization regions of the optimal quantizer are difficult to characterize since the optimal quantizer (and hence the number of quantization regions together with their locations and volumes) changes with $D$. To sidestep this problem, we replace (33) by a slightly more sophisticated upper bound on $h\left(\mathbf{X} \mid \hat{\mathbf{X}}=\hat{\mathbf{x}}_{i}\right)$.

\section{A. Entropy Bounds}

Recall that (33) follows from the fact that the distribution of support $\mathcal{S}_{i}$ that maximizes differential entropy is the uniform distribution on $\mathcal{S}_{i}$. This result, in turn, is a direct consequence of the nonnegativity of relative entropy. Indeed, let $D(P \| Q)$ denote the relative entropy between two probability distributions $P$ and $Q$, i.e.,

$$
D(P \| Q) \triangleq \begin{cases}\int \log \frac{\mathrm{d} P(x)}{\mathrm{d} Q(x)} \mathrm{d} P(x), & \text { if } P \ll Q \\ \infty, & \text { otherwise }\end{cases}
$$

where we use the notation $P \ll Q$ to indicate that $P$ is absolutely continuous with respect to $Q$. Relative entropy satisfies $D(P \| Q) \geq 0$ with equality if, and only if, $P$ and $Q$ coincide. For general probability distributions $P$ and $Q$, this follows from the classic work of Dobrushin, Gelfand, Yaglom, and Perez; see [22, Chapter 2] and references therein. For the case where $P$ and $Q$ are absolutely continuous with respect to each other, i.e., $P \ll Q$ and $Q \ll P$, this was demonstrated by Kullback and Leibler [23, Lemma 3.1]. For the case where both $P$ and $Q$ are (discrete) probability mass functions, the nonnegativity of relative entropy is sometimes attributed to the mathematical physicist Josiah Willard Gibbs (1839-1903). Using the nonnegativity of relative entropy, we can recover the fact that the distribution of support $\mathcal{S}_{i}$ that maximizes differential entropy is the uniform distribution on $\mathcal{S}_{i}$ as follows:

Let $Q$ be the uniform distribution on $\mathcal{S}_{i}$, so its pdf is equal to $u(x)=1 / \Delta_{i} \mathbf{1}\left\{x \in \mathcal{S}_{i}\right\}, x \in \mathbb{R}$. Further let $P$ be absolutely 
continuous with respect to the Lebesgue measure, and denote its pdf by $f$. Assume that $P$ has support $\mathcal{S}_{i}$. Evaluating the relative entropy for these distributions, we obtain

$$
D(P \| Q)=\int_{\mathcal{S}_{i}} f(x) \log \frac{f(x)}{u(x)} \mathrm{d} x=-h(X)+\log \Delta_{i}
$$

where $X$ is a random variable with pdf $f$. By the nonnegativity of relative entropy, we thus have that $h(X) \leq \log \Delta_{i}$. The entropy-maximizing property of uniform distributions follows because $\log \Delta_{i}$ is the differential entropy of a random variable that is uniformly distributed on $\mathcal{S}_{i}$.

In general, by choosing $P$ and $Q$ to be absolutely continuous with respect to the Lebesgue measure, and by denoting their pdfs by $f$ and $g$, respectively, we can upper-bound the differential entropy of a random vector $\mathbf{X}$ with pdf $f$ by

$$
h(\mathbf{X}) \leq-\int f(\mathbf{x}) \log g(\mathbf{x}) \mathrm{d} \mathbf{x} .
$$

This inequality holds for any arbitrary pdf $g$, and it is tight if $f(\mathbf{x})=g(\mathbf{x})$ almost everywhere. It is therefore a useful tool to upper-bound differential entropies that are difficult to evaluate in closed form. For future reference, we summarize the entropy inequality in the following lemma.

Lemma 3: Let $f$ and $g$ be arbitrary pdfs. If $-\int f(\mathbf{x}) \log f(\mathbf{x}) \mathrm{d} \mathbf{x}$ is finite, then $-\int f(\mathbf{x}) \log g(\mathbf{x}) \mathrm{d} \mathbf{x}$ exists and

$$
-\int f(\mathbf{x}) \log f(\mathbf{x}) \mathrm{d} \mathbf{x} \leq-\int f(\mathbf{x}) \log g(\mathbf{x}) \mathrm{d} \mathbf{x}
$$

with equality if, and only if, $f(\mathbf{x})=g(\mathbf{x})$ almost everywhere.

Proof: The lemma appears in this form in [24, Lemma 8.3.1]. Its proof is based on the nonnegativity of relative entropy.

The approach of upper-bounding differential entropy by replacing the true pdf $f$ by an auxiliary pdf $g$ is reminiscent of a technique proposed in [25] to derive upper bounds on channel capacity. Specifically, the mutual information between a channel input $\mathbf{X}$ and a channel output $\mathbf{Y}$ is upper-bounded by [25, Theorem 5.1]

$$
I(\mathbf{X} ; \mathbf{Y}) \leq \int D(W(\cdot \mid \mathbf{x}) \| R(\cdot)) \mathrm{d} Q(\mathbf{x})
$$

where $W(\cdot \mid \cdot)$ denotes the conditional distribution of the channel output $\mathbf{Y}$ given its input $\mathbf{X}, Q$ denotes the distribution of $\mathbf{X}$, and $R$ denotes an arbitrary distribution on the output alphabet (not necessarily the one induced by $Q$ and $W$ ). For channels with finite input and output alphabets, this inequality follows by Topsøe's identity [26]; see also [27, Problem 8.1]. When the conditional distribution of $\mathbf{Y}$ given $\mathbf{X}$ is absolutely continuous with respect to the Lebesgue measure, the inequality (49) is equivalent to (48). Any output distribution $R$ applied to (49) yields an upper bound on the mutual information. Inter alia, this approach has been followed to derive upper bounds on the capacity of noncoherent fading channels [25], [28][30], phase-noise channels [31]-[33], the Poisson channel [34]-[36], optical intensity channels [37], [38], the additive inverse Gaussian noise channel [39], channels that heat up [40], or channels whose inputs have bounded support [41].

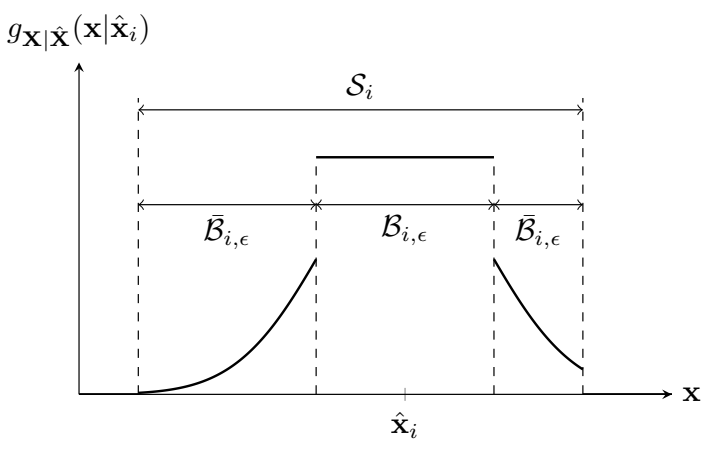

Figure 1. Example of the conditional pdf $g_{\mathbf{X} \mid \hat{\mathbf{X}}}$, as defined in (50), for a one-dimensional source and squared-error distortion. While in this specific example the quantization region $\mathcal{S}_{i}$ is an interval and $\hat{\mathbf{x}}_{i} \in \mathcal{S}_{i}$, recall that in general $\hat{\mathbf{x}}_{i}$ and $\mathcal{S}_{i}$ can be arbitrary.

\section{B. Auxiliary Probability Density Function}

In order to upper-bound the conditional differential entropy $h\left(\mathbf{X} \mid \hat{\mathbf{X}}=\hat{\mathbf{x}}_{i}\right)$, we apply Lemma 3 with the conditional pdf

$$
g_{\mathbf{X} \mid \hat{\mathbf{X}}}\left(\mathbf{x} \mid \hat{\mathbf{x}}_{i}\right)= \begin{cases}\frac{1}{\mathrm{~K}_{i, \epsilon}}, & \mathbf{x} \in \mathcal{B}_{i, \epsilon} \\ \frac{1}{\mathrm{~K}_{i, \epsilon}} \frac{r}{\delta^{d / r}} e^{-\frac{\left\|\mathbf{x}-\hat{\mathbf{x}}_{i}\right\|^{r}}{D \delta}}, & \mathbf{x} \in \overline{\mathcal{B}}_{i, \epsilon}\end{cases}
$$

where $\delta$ and $\epsilon$ are parameters to be specified later, and

$$
\begin{aligned}
& \mathcal{B}_{i, \epsilon} \triangleq\left\{\mathbf{x} \in \mathcal{S}_{i}:\left\|\mathbf{x}-\hat{\mathbf{x}}_{i}\right\| \leq \epsilon\right\} \\
& \overline{\mathcal{B}}_{i, \epsilon} \triangleq\left\{\mathbf{x} \in \mathcal{S}_{i}:\left\|\mathbf{x}-\hat{\mathbf{x}}_{i}\right\|>\epsilon\right\} \\
& \mathrm{K}_{i, \epsilon} \triangleq \Lambda_{i, \epsilon}+\frac{r}{\delta^{d / r}} \int_{\overline{\mathcal{B}}_{i, \epsilon}} e^{-\frac{\left\|\mathbf{x}-\hat{\mathbf{x}}_{i}\right\|^{r}}{D \delta}} \mathrm{d} \mathbf{x} .
\end{aligned}
$$

In (51c), $\Lambda_{i, \epsilon}$ denotes the Lebesgue measure of $\mathcal{B}_{i, \epsilon}$. To simplify notation, we denote the probability of $\mathbf{X}$ being in $\mathcal{B}_{i, \epsilon}$ by $p_{i, \epsilon}$, and the probability of $\mathbf{X}$ being in $\overline{\mathcal{B}}_{i, \epsilon}$ by $\bar{p}_{i, \epsilon}$.

The conditional pdf of $\mathbf{X}$ given $\hat{\mathbf{X}}$, as defined in (50), is uniform on a set of measure $\Lambda_{i, \epsilon}$ around $\hat{\mathbf{x}}_{i}$ and then decays exponentially. An example of $g_{\mathbf{X} \mid \hat{\mathbf{X}}}$ is illustrated in Figure 1. Intuitively, if $\epsilon^{d}$ decays more slowly than $\Delta_{i}$ as $D$ tends to zero, then with high probability $\mathbf{X}$ lies in $\mathcal{B}_{i, \epsilon}$ and the upper bound obtained from Lemma 3 is essentially equivalent to (33) but with $\Delta_{i}$ replaced by $\Lambda_{i, \epsilon}$. Our choice of $g_{\mathbf{X} \mid \hat{\mathbf{X}}}$ for $\mathbf{x} \in \overline{\mathcal{B}}_{i, \epsilon}$ allows us to control the contribution of $\mathbf{X}$ 's lying outside of $\mathcal{B}_{i, \epsilon}$. We then need to show that

$$
\varlimsup_{D \downarrow 0} \frac{1}{D} \sum_{i} p_{i, \epsilon} \Lambda_{i, \epsilon}^{r} \leq V_{d}^{r / d}\left(1+\frac{r}{d}\right)
$$

which corresponds to (37) generalized to arbitrary $d$ and $r$, but with $\Delta_{i}$ replaced by $\Lambda_{i, \epsilon}$ and with $p_{i}$ replaced by $p_{i, \epsilon}$. By construction of $\mathcal{B}_{i, \epsilon}$, we have that $\left\|\mathbf{x}-\hat{\mathbf{x}}_{i}\right\|^{r} \leq \epsilon^{r}$, so $\mathcal{B}_{i, \epsilon}$ satisfies (31) upon choosing $\epsilon^{r}=D / \kappa$ (for some constant $\kappa)$. The claim (52) follows therefore from the steps (38)-(44). Thus, by using Lemma 3 together with (50), we can replace $\Delta_{i}$ (whose behavior as a function of $D$ is unknown) by $\Lambda_{i, \epsilon}$ (whose behavior can be controlled by cleverly choosing $\epsilon$ ).

We next provide a number of auxiliary results that we shall need throughout the proof. The proof of Theorem 1 is then given in Section IV-C. 
Lemma 4: The normalizing constant $\mathrm{K}_{i, \epsilon}$ satisfies

$$
\begin{aligned}
\mathrm{K}_{i, \epsilon} & \leq \Lambda_{i, \epsilon}+d V_{d} D^{d / r} \Gamma\left(\frac{d}{r}, \frac{\epsilon^{r}}{D \delta}\right) \\
& \leq \epsilon^{d} V_{d}+d V_{d} D^{d / r} \Gamma\left(\frac{d}{r}\right)
\end{aligned}
$$

where

$$
\Gamma(\alpha, x) \triangleq \int_{x}^{\infty} e^{-t} t^{\alpha-1} \mathrm{~d} t, \quad(\alpha>0, x \geq 0)
$$

denotes the upper incomplete Gamma function.

Proof: The first inequality (53a) follows from the definition of $\mathrm{K}_{i, \epsilon},(51 \mathrm{c})$, and by upper-bounding the integral on the RHS of (51c). Indeed, since $\overline{\mathcal{B}}_{i, \epsilon} \subseteq\left\{\mathbf{x} \in \mathbb{R}^{d}:\left\|\mathbf{x}-\hat{\mathbf{x}}_{i}\right\|>\epsilon\right\}$, we have

$$
\begin{aligned}
\frac{r}{\delta^{d / r}} \int_{\overline{\mathcal{B}}_{i, \epsilon}} e^{-\frac{\left\|\mathbf{x}-\hat{\mathbf{x}}_{i}\right\|^{r}}{D \delta}} \mathrm{d} \mathbf{x} & \leq \frac{r}{\delta^{d / r}} \int_{\left\|\mathbf{x}-\hat{\mathbf{x}}_{i}\right\|>\epsilon} e^{-\frac{\left\|\mathbf{x}-\hat{\mathbf{x}}_{i}\right\|^{r}}{D \delta}} \mathrm{d} \mathbf{x} \\
& =d V_{d} \frac{r}{\delta^{d / r}} \int_{\rho>\epsilon} \rho^{d-1} e^{-\frac{\rho^{r}}{D \delta}} \mathrm{d} \rho \\
& =d V_{d} D^{d / r} \int_{\xi>\frac{\epsilon^{r}}{D \delta}} \xi^{d / r-1} e^{-\xi} \mathrm{d} \xi \\
& =d V_{d} D^{d / r} \Gamma\left(\frac{d}{r}, \frac{\epsilon^{r}}{D \delta}\right)
\end{aligned}
$$

where the second step follows by writing $\mathbf{x}-\hat{\mathbf{x}}_{i}$ in polar coordinates and by using that the surface area of the $d$ dimensional ball of radius $\rho=\left\|\mathbf{x}-\hat{\mathbf{x}}_{i}\right\|$ is $d V_{d} \rho^{d-1}$ (see, e.g., [8, eq. (10)]), and the third step follows by the change of variable $\xi=\rho^{r} /(D \delta)$.

The second inequality (53b) follows by upper-bounding

$$
\Lambda_{i, \epsilon} \leq \int_{\|\mathbf{x}-\hat{\mathbf{x}}\| \leq \epsilon} \mathrm{dx}=\epsilon^{d} V_{d}
$$

and because $\Gamma(d / r, x) \leq \Gamma(d / r), x \geq 0$.

Lemma 5: The set $\overline{\mathcal{B}}_{i, \epsilon}$ satisfies

$$
\begin{array}{r}
\sum_{i} \bar{p}_{i, \epsilon} \leq \frac{D}{\epsilon^{r}} \\
\sum_{i} \mathrm{E}\left[\left\|\mathbf{X}-\hat{\mathbf{x}}_{i}\right\|^{r} \mathbf{1}\left\{\mathbf{X} \in \overline{\mathcal{B}}_{i, \epsilon}\right\}\right] \leq D .
\end{array}
$$

Proof: We first prove (57a). By the distortion constraint (2), and since $\overline{\mathcal{B}}_{i, \epsilon} \subseteq \mathcal{S}_{i}$ and $\left\|\mathbf{x}-\hat{\mathbf{x}}_{i}\right\|>\epsilon$ for $\mathbf{x} \in \overline{\mathcal{B}}_{i, \epsilon}$, we have

$$
\begin{aligned}
D & \geq \sum_{i} \int_{\mathcal{S}_{i}} f_{\mathbf{X}}(\mathbf{x})\left\|\mathbf{x}-\hat{\mathbf{x}}_{i}\right\|^{r} \mathrm{~d} \mathbf{x} \\
& \geq \sum_{i} \int_{\overline{\mathcal{B}}_{i, \epsilon}} f_{\mathbf{X}}(\mathbf{x})\left\|\mathbf{x}-\hat{\mathbf{x}}_{i}\right\|^{r} \mathrm{~d} \mathbf{x} \\
& \geq \sum_{i} \int_{\overline{\mathcal{B}}_{i, \epsilon}} f_{\mathbf{X}}(\mathbf{x}) \epsilon^{r} \mathrm{~d} \mathbf{x} .
\end{aligned}
$$

Using that $\epsilon$ neither depends on $i$ nor on $\mathbf{x}$, (57a) follows by dividing both sides of (58) by $\epsilon^{r}$.
To prove (57b) we use again that $\overline{\mathcal{B}}_{i, \epsilon} \subseteq \mathcal{S}_{i}$ to obtain

$$
\begin{aligned}
& \sum_{i} \mathrm{E}\left[\left\|\mathbf{X}-\hat{\mathbf{x}}_{i}\right\|^{r} \mathbf{1}\left\{\mathbf{X} \in \overline{\mathcal{B}}_{i, \epsilon}\right\}\right] \\
& \quad=\sum_{i} \int_{\overline{\mathcal{B}}_{i, \epsilon}} f_{\mathbf{X}}(\mathbf{x})\left\|\mathbf{x}-\hat{\mathbf{x}}_{i}\right\|^{r} \mathrm{~d} \mathbf{x} \\
& \quad \leq \mathrm{E}\left[\|\mathbf{X}-\hat{\mathbf{X}}\|^{r}\right] .
\end{aligned}
$$

By the distortion constraint (2), this yields (57b).

\section{Proof of Theorem 1}

Expanding $I(\mathbf{X} ; \hat{\mathbf{X}})$ as $h(\mathbf{X})-h(\mathbf{X} \mid \hat{\mathbf{X}})$, we obtain from (5) and (16) that the asymptotic excess rate can be expressed as

$$
\begin{aligned}
\mathrm{R}_{r, d}= & \frac{d}{r} \log \left(\frac{r}{d}\left(V_{d} \Gamma(1+d / r)\right)^{r / d} e\right) \\
& -\varlimsup_{D \downarrow 0}\left\{\sup _{q(\cdot)} h(\mathbf{X} \mid \hat{\mathbf{X}})-\frac{d}{r} \log D\right\} .
\end{aligned}
$$

To derive the lower bound (22) given in Theorem 1, it remains to show that

$$
\varlimsup_{D \downarrow 0}\left\{\sup _{q(\cdot)} h(\mathbf{X} \mid \hat{\mathbf{X}})-\frac{d}{r} \log D\right\} \leq \frac{d}{r} \log \left(V_{d}^{r / d}(1+r / d)\right) .
$$

To this end, we upper-bound the conditional differential entropy $h(\mathbf{X} \mid \hat{\mathbf{X}})$ using Lemma 3 together with (50). This yields for every $\hat{\mathbf{X}}=\hat{\mathbf{x}}_{i}$

$$
\begin{aligned}
h(\mathbf{X} \mid & \left.\hat{\mathbf{X}}=\hat{\mathbf{x}}_{i}\right) \\
\leq & \log \mathrm{K}_{i, \epsilon} \\
& \quad-\mathrm{E}\left[\log \left(\frac{r}{\delta^{d / r}} e^{-\frac{\left\|\mathbf{X}-\hat{\mathbf{x}}_{i}\right\|^{r}}{D \delta}}\right) \mathbf{1}\left\{\mathbf{X} \in \overline{\mathcal{B}}_{i, \epsilon}\right\} \mid \mathbf{X} \in \mathcal{S}_{i}\right] \\
\leq & \log \left(\Lambda_{i, \epsilon}+d V_{d} D^{d / r} \Gamma\left(\frac{d}{r}, \frac{\epsilon^{r}}{D \delta}\right)\right) \\
\quad & \left|\log \left(\frac{r}{\delta^{d / r}}\right)\right| \operatorname{Pr}\left(\mathbf{X} \in \overline{\mathcal{B}}_{i, \epsilon} \mid \mathbf{X} \in \mathcal{S}_{i}\right) \\
& +\frac{1}{D \delta} \mathrm{E}\left[\left\|\mathbf{X}-\hat{\mathbf{x}}_{i}\right\|^{r} \mathbf{1}\left\{\mathbf{X} \in \overline{\mathcal{B}}_{i, \epsilon}\right\} \mid \mathbf{X} \in \mathcal{S}_{i}\right]
\end{aligned}
$$

where the second inequality follows from inequality (53a) in Lemma 4 and because $-\log \left(r / \delta^{d / r}\right) \leq\left|\log \left(r / \delta^{d / r}\right)\right|$. Averaging over $\hat{\mathbf{X}}$ then yields

$$
\begin{aligned}
h(\mathbf{X} \mid \hat{\mathbf{X}}) \leq & \sum_{i} p_{i} \log \left(\Lambda_{i, \epsilon}+d V_{d} D^{d / r} \Gamma\left(\frac{d}{r}, \frac{\epsilon^{r}}{D \delta}\right)\right) \\
& +\left|\log \left(\frac{r}{\delta^{d / r}}\right)\right| \sum_{i} \bar{p}_{i, \epsilon} \\
& +\frac{1}{D \delta} \sum_{i} \mathbf{E}\left[\left\|\mathbf{X}-\hat{\mathbf{x}}_{i}\right\|^{r} \mathbf{1}\left\{\mathbf{X} \in \overline{\mathcal{B}}_{i, \epsilon}\right\}\right] .
\end{aligned}
$$

By Lemma 5, this can be further upper-bounded by

$$
\begin{aligned}
h(\mathbf{X} \mid \hat{\mathbf{X}}) \leq & \sum_{i} p_{i} \log \left(\Lambda_{i, \epsilon}+d V_{d} D^{d / r} \Gamma\left(\frac{d}{r}, \frac{\epsilon^{r}}{D \delta}\right)\right) \\
& +\left|\log \frac{r}{\delta^{d / r}}\right| \frac{D}{\epsilon^{r}}+\frac{1}{\delta} .
\end{aligned}
$$

We next choose

$$
\epsilon^{r}=\frac{D}{\kappa}
$$


for some $\kappa>0$ that we will let tend to zero at the end of the proof. For ease of exposition, we do not always make this choice explicit in the notation but write $\epsilon^{r}$ or $D / \kappa$ depending on which is more convenient.

With this choice, the second term on the RHS of (64) becomes $\kappa\left|\log \left(r / \delta^{d / r}\right)\right|$. To evaluate the first term on the RHS of (64), we express $p_{i}$ as

$$
p_{i}=p_{i, \epsilon}+\bar{p}_{i, \epsilon}
$$

and define

$$
\wp_{\epsilon} \triangleq \sum_{i} \bar{p}_{i, \epsilon}
$$

By Lemma 5, we have

$$
\wp_{\epsilon} \leq \kappa
$$

which vanishes as we let $\kappa$ tend to zero. With the above definition, and applying inequality (53b) in Lemma 4, we obtain for the first term on the RHS of (64) that

$$
\begin{aligned}
\sum_{i} & p_{i} \log \left(\Lambda_{i, \epsilon}+d V_{d} D^{d / r} \Gamma\left(\frac{d}{r}, \frac{\epsilon^{r}}{D \delta}\right)\right) \\
= & \sum_{i} p_{i, \epsilon} \log \left(\Lambda_{i, \epsilon}+d V_{d} D^{d / r} \Gamma\left(\frac{d}{r}, \frac{1}{\kappa \delta}\right)\right) \\
& +\sum_{i} \bar{p}_{i, \epsilon} \log \left(\Lambda_{i, \epsilon}+d V_{d} D^{d / r} \Gamma\left(\frac{d}{r}, \frac{1}{\kappa \delta}\right)\right) \\
\leq & \sum_{i} p_{i, \epsilon} \log \left(\Lambda_{i, \epsilon}+d V_{d} D^{d / r} \Gamma\left(\frac{d}{r}, \frac{1}{\kappa \delta}\right)\right) \\
& +\wp_{\epsilon} \log \left(V_{d} \frac{D^{d / r}}{\kappa^{d / r}}+d V_{d} D^{d / r} \Gamma(d / r)\right) .
\end{aligned}
$$

Using (68) and that $\sum_{i} p_{i, \epsilon}+\wp_{\epsilon}=1$, (69) becomes

$$
\begin{aligned}
\sum_{i} & p_{i} \log \left(\Lambda_{i, \epsilon}+d V_{d} D^{d / r} \Gamma\left(\frac{d}{r}, \frac{1}{\kappa \delta}\right)\right) \\
\leq & \sum_{i} p_{i, \epsilon} \log \left(\frac{\Lambda_{i, \epsilon}}{D^{d / r}}+d V_{d} \Gamma\left(\frac{d}{r}, \frac{1}{\kappa \delta}\right)\right) \\
& +\wp_{\epsilon} \log \left(\frac{V_{d}}{\kappa^{d / r}}+d V_{d} \Gamma(d / r)\right)+\frac{d}{r} \log D \\
\leq & \frac{d}{r} \sum_{i} p_{i, \epsilon} \log \left(\frac{\Lambda_{i, \epsilon}}{D^{d / r}}+d V_{d} \Gamma\left(\frac{d}{r}, \frac{1}{\kappa \delta}\right)\right)^{r / d} \\
& +\kappa \log \left(\frac{V_{d}}{\kappa^{d / r}}+d V_{d} \Gamma(d / r)\right)+\frac{d}{r} \log D .
\end{aligned}
$$

By Jensen's inequality,

$$
\begin{aligned}
& \sum_{i} \frac{p_{i, \epsilon}}{1-\wp_{\epsilon}} \log \left(\frac{\Lambda_{i, \epsilon}}{D^{d / r}}+d V_{d} \Gamma\left(\frac{d}{r}, \frac{1}{\kappa \delta}\right)\right)^{r / d} \\
& \quad \leq \log \left(\sum_{i} \frac{p_{i, \epsilon}}{1-\wp_{\epsilon}}\left[\frac{\Lambda_{i, \epsilon}}{D^{d / r}}+d V_{d} \Gamma\left(\frac{d}{r}, \frac{1}{\kappa \delta}\right)\right]^{r / d}\right) .
\end{aligned}
$$

For $r / d<1$, we have $(x+\alpha)^{r / d} \leq x^{r / d}+\alpha^{r / d}$ for every $x, \alpha \geq 0$, so in this case

$$
\begin{aligned}
& \sum_{i} \frac{p_{i, \epsilon}}{1-\wp_{\epsilon}}\left[\frac{\Lambda_{i, \epsilon}}{D^{d / r}}+d V_{d} \Gamma\left(\frac{d}{r}, \frac{1}{\kappa \delta}\right)\right]^{r / d} \\
& \quad \leq \sum_{i} \frac{p_{i, \epsilon}}{1-\wp_{\epsilon}} \frac{\Lambda_{i, \epsilon}^{r / d}}{D}+d^{r / d} V_{d}^{r / d} \Gamma\left(\frac{d}{r}, \frac{1}{\kappa \delta}\right)^{r / d} .
\end{aligned}
$$

Similarly, for $r / d \geq 1$, the function $x \mapsto\left(x^{d / r}+\alpha\right)^{r / d}$ is concave for every $\alpha \geq 0$, so by Jensen's inequality

$$
\begin{aligned}
\sum_{i} & \frac{p_{i, \epsilon}}{1-\wp_{\epsilon}}\left[\frac{\Lambda_{i, \epsilon}}{D^{d / r}}+d V_{d} \Gamma\left(\frac{d}{r}, \frac{1}{\kappa \delta}\right)\right]^{r / d} \\
\leq & {\left[\left(\sum_{i} \frac{p_{i, \epsilon}}{1-\wp_{\epsilon}} \frac{\Lambda_{i, \epsilon}^{r / d}}{D}\right)^{d / r}+d V_{d} \Gamma\left(\frac{d}{r}, \frac{1}{\kappa \delta}\right)\right]^{r / d} . }
\end{aligned}
$$

We next generalize (37), namely,

$$
\varlimsup_{D \downarrow 0} \frac{1}{D} \sum_{i} p_{i} \Delta_{i}^{2} \leq 12
$$

to the $d$-dimensional sets $\mathcal{B}_{i, \epsilon}$ of Lebesgue measure $\Lambda_{i, \epsilon}$. To this end, we follow essentially the steps (38)-(44) in Section III with $\mathcal{S}_{i}$ replaced by $\mathcal{B}_{i, \epsilon}$ and with $\Delta_{i}$ replaced by $\Lambda_{i, \epsilon}$. However, (43) is based on Lebesgue's differentiation theorem, which requires that the families of sets $\mathcal{B}_{i, \epsilon}$ (parametrized by $D)$ have bounded eccentricity. ${ }^{4}$ Since $\mathcal{B}_{i, \epsilon}$ is the intersection of $\mathcal{S}_{i}$ with the $d$-dimensional ball of radius $\epsilon$ centered at $\hat{\mathbf{x}}_{i}$, cf. (51a), and since $\mathcal{S}_{i}$ is arbitrary, the sets $\mathcal{B}_{i, \epsilon}$ may not satisfy this condition. In the one-dimensional case, a sufficient condition for $\mathcal{B}_{i, \epsilon}$ having bounded eccentricity would be that, for every distortion $D$, the quantization regions $\mathcal{S}_{i}$ are convex. This in turn can be assumed without loss of optimality, e.g., for squared-error distortion and sources with well-behaved pdfs [16]. However, for one-dimensional sources with general pdfs, or for higher-dimensional sources, assuming convex quantization regions may be too restrictive. Fortunately, the families of sets $\mathcal{B}_{i, \epsilon}$ that have not bounded eccentricity can be disregarded without affecting the final result. The inequality (37) can therefore be generalized to the case at hand without imposing any additional constraints on the quantization regions $\left\{\mathcal{S}_{i}\right\}$ or the source pdf $f_{\mathbf{X}}$. The result is stated in the following lemma.

Lemma 6: Let the sets $\left\{\mathcal{B}_{i, \epsilon}\right\}$ be defined in (51a), and let $\left\{\Lambda_{i, \epsilon}\right\}$ denote the Lebesgue measures of these sets. Assume that $\epsilon^{r}=D / \kappa$. Then, for every $\kappa>0$,

$$
\varlimsup_{D \downarrow 0} \sup _{q(\cdot)} \sum_{i} p_{i, \epsilon} \frac{\Lambda_{i, \epsilon}^{r / d}}{D} \leq V_{d}^{r / d}\left(1+\frac{r}{d}\right) .
$$

Proof: See Appendix A.

\footnotetext{
${ }^{4} \mathrm{~A}$ family $\mathcal{F}$ of sets is said to have bounded eccentricity if there exists a constant $c>0$ such that for every $\mathcal{S} \in \mathcal{F}$ the Lebesgue measure of $\mathcal{S}$ is not smaller than $c$ times the volume of the smallest ball containing $\mathcal{S}$.
} 
Combining Lemma 6 with (64)-(72), and using $0 \leq \wp_{\epsilon} \leq \kappa$, we obtain that

$$
\begin{aligned}
& \varlimsup_{D \downarrow 0}\left\{\sup _{q(\cdot)} h(\mathbf{X} \mid \hat{\mathbf{X}})-\frac{d}{r} \log D\right\} \\
& \leq \frac{d}{r} \log \left(\frac{V_{d}^{r / d}(1+r / d)}{1-\kappa}+d^{r / d} V_{d}^{r / d} \Gamma\left(\frac{d}{r}, \frac{1}{\kappa \delta}\right)^{r / d}\right) \\
&+\kappa \log \left(\frac{V_{d}}{\kappa^{d / r}}+d V_{d} \Gamma(d / r)\right)+\kappa\left|\log \frac{r}{\delta^{d / r}}\right|+\frac{1}{\delta}
\end{aligned}
$$

for $r / d<1$, and

$$
\begin{aligned}
& \varlimsup_{D \downarrow 0}\left\{\sup _{q(\cdot)} h(\mathbf{X} \mid \hat{\mathbf{X}})-\frac{d}{r} \log D\right\} \\
& \leq \log \left(\frac{V_{d}(1+r / d)^{d / r}}{(1-\kappa)^{d / r}}+d V_{d} \Gamma\left(\frac{d}{r}, \frac{1}{\kappa \delta}\right)\right) \\
& \quad+\kappa \log \left(\frac{V_{d}}{\kappa^{d / r}}+d V_{d} \Gamma(d / r)\right)+\kappa\left|\log \frac{r}{\delta^{d / r}}\right|+\frac{1}{\delta}
\end{aligned}
$$

for $r / d \geq 1$. Using that $\lim _{\xi \rightarrow \infty} \Gamma(d / r, \xi)=0$ and $\lim _{\xi \rightarrow 0} \xi \log \left(\alpha / \xi^{d / r}+\beta\right)=0$ (for any $\alpha, \beta>0$ ), letting $\kappa \rightarrow 0$ yields

$$
\begin{aligned}
\varlimsup_{D \downarrow 0}\left\{\sup _{q(\cdot)} h(\mathbf{X} \mid \hat{\mathbf{X}})-\right. & \left.\frac{d}{r} \log D\right\} \\
& \leq \frac{d}{r} \log \left(V_{d}^{r / d}(1+r / d)\right)+\frac{1}{\delta} .
\end{aligned}
$$

This in turn proves (61) upon letting $\delta \rightarrow \infty$ and concludes the proof of Theorem 1 .

\section{Asymptotically Optimal Quantizers}

As mentioned at the end of Section II, in the onedimensional case uniform quantizers achieve the asymptotic excess rate $\mathrm{R}_{r, 1}$. Hence, uniform quantizers are asymptotically optimal as the allowed distortion tends to zero. One may wonder whether every sequence of quantizers achieving $\mathrm{R}_{r, 1}$ must converge to a uniform quantizer as $D \rightarrow 0$, or whether uniform quantizers are merely a convenient choice and other quantizers with vanishing cells are also asymptotically optimal. In this section, we partially address this question by presenting in Theorem 7 a necessary condition for the asymptotic optimality of a sequence of quantizers (parametrized by $D$ ). We then apply this condition to the family of almost-regular quantizers.

Theorem 7: Let the one-dimensional source $X$ have a pdf, and assume that $h(X)$ and $H(\lfloor X\rfloor)$ are finite. Suppose the sequence of quantizers $q(\cdot)$ (parametrized by $D$ ) satisfying the distortion constraint $\mathrm{E}\left[|X-q(X)|^{r}\right] \leq D$ achieves the asymptotic excess rate

$$
\varliminf_{D \downarrow 0}\{H(q(X))-R(D)\}=\frac{1}{r} \log \left(\frac{\Gamma(1+1 / r)^{r} e}{1+1 / r}\right) .
$$

Then,

$$
\lim _{\rho \rightarrow \infty} \varlimsup_{D \downarrow 0} \sum_{i} p_{i} \mathbf{1}\left\{\left|\frac{\Lambda_{i, \rho D^{1 / r}}^{r}}{D}-V_{1}^{r}(1+r)\right| \leq \vartheta\right\}=1
$$

for every $\vartheta>0$. Here, $\Lambda_{i, \rho D^{1 / r}}$ denotes the Lebesgue measure of $\mathcal{B}_{i, \epsilon}$, defined in (51a), for $\epsilon=\rho D^{1 / r}$.
Proof: This result is a direct consequence of Jensen's inequality applied in (71) in the proof of Theorem 1. See Appendix B for a detailed proof.

Defining the function

$$
\Lambda_{\rho D^{1 / r}}(x) \triangleq \sum_{i} \Lambda_{i, \rho D^{1 / r}} \mathbf{1}\left\{x \in \mathcal{S}_{i}\right\}, \quad x \in \mathbb{R}
$$

the necessary condition (78) can be written as

$$
\lim _{\rho \rightarrow \infty} \varlimsup_{D \downarrow 0} \operatorname{Pr}\left(\left|\frac{\Lambda_{\rho D^{1 / r}}^{r}(X)}{D}-V_{1}^{r}(1+r)\right| \leq \vartheta\right)=1
$$

for every $\vartheta>0$. Theorem 7 can thus be paraphrased as follows: "A sequence of quantizers achieves the asymptotic excess rate (77) only if $\Lambda_{\rho D^{1 / r}}^{r}(X) / D$ converges in probability to $V_{1}^{r}(1+r)$ as $D \downarrow 0$ and $\rho \rightarrow \infty$."

A quantizer $q(\cdot)$ is said to be almost regular if there exists a set $\overline{\mathcal{S}} \subset \mathcal{X}$ of Lebesgue measure zero such that on $\mathcal{X} \backslash \overline{\mathcal{S}}$ the quantization regions are intervals containing the reconstruction value [16]. (For all $x \in \overline{\mathcal{S}}$, we can define $q(x)$ in an arbitrary manner without changing the entropy and distortion of $q(\cdot)$.) In other words, an almost-regular quantizer $q(\cdot)$ can be written as

$$
\begin{array}{ll}
q(x)=\sum_{i} c_{i} \mathbf{1}\left\{a_{i} \leq x<b_{i}\right\}, & \text { for } x \in \mathcal{X} \backslash \overline{\mathcal{S}} \\
q(x)=\sum_{i} \bar{x}_{i} \mathbf{1}\left\{x \in \overline{\mathcal{S}}_{i}\right\}, & \text { for } x \in \overline{\mathcal{S}}
\end{array}
$$

where $a_{i} \leq c_{i}<b_{i}$, and where $\bar{x}_{i}$ and $\overline{\mathcal{S}}_{i}$ are arbitrary.

For almost-regular quantizers, condition (78) in Theorem 7 can be simplified as follows. Firstly, since the source has a pdf and $\overline{\mathcal{S}}$ has measure zero,

$$
\sum_{i} \operatorname{Pr}\left(X \in \mathcal{S}_{i} \cap \overline{\mathcal{S}}\right)=0 .
$$

Secondly, for any quantization region $\left[a_{i}, b_{i}\right) \subseteq \mathcal{X} \backslash \overline{\mathcal{S}}$ and reconstruction value $c_{i} \in\left[a_{i}, b_{i}\right)$, we have

$$
\min \left\{\Delta_{i}^{r}, \rho^{r} D\right\} \leq \Lambda_{i, \rho D^{1 / r}}^{r} \leq \Delta_{i}^{r}
$$

where $\Delta_{i}=b_{i}-a_{i}$. Thus, if $\rho$ is large, then either $\Delta_{i}$ is large, too, or $\Lambda_{i, \rho D^{1 / r}}=\Delta_{i}$. Indeed, suppose $\rho^{r} \geq V_{1}^{r}(1+r)+\vartheta$. If

$$
\frac{\Lambda_{i, \rho D^{1 / r}}^{r}}{D} \leq V_{1}^{r}(1+r)+\vartheta
$$

then it must hold that $\min \left\{\Delta_{i}^{r}, \rho^{r} D\right\}=\Delta_{i}^{r}$, since otherwise (83) would be violated. It then follows by (83) that $\Lambda_{i, \rho D^{1 / r}}$ is equal to $\Delta_{i}$. In contrast, if

$$
\frac{\Lambda_{i, \rho D^{1 / r}}^{r}}{D}>V_{1}^{r}(1+r)+\vartheta
$$

then by the right-most inequality in (83),

$$
\frac{\Delta_{i}^{r}}{D}>V_{1}^{r}(1+r)+\vartheta
$$

Consequently, for any quantization region $\left[a_{i}, b_{i}\right) \subseteq \mathcal{X} \backslash \overline{\mathcal{S}}$ and reconstruction value $c_{i} \in\left[a_{1}, b_{i}\right)$, we obtain that

$$
\begin{aligned}
\mathbf{1}\left\{\left|\frac{\Lambda_{i, \rho D^{1 / r}}^{r}}{D}-V_{1}^{r}(1+r)\right| \leq \vartheta\right\} \\
=1\left\{\left|\frac{\Delta_{i}^{r}}{D}-V_{1}^{r}(1+r)\right| \leq \vartheta\right\}
\end{aligned}
$$


for $\rho \geq\left(V_{1}^{r}(1+r)+\vartheta\right)^{1 / r}$. We thus have the following result:

Corollary 8: Let the one-dimensional source $X$ have a pdf, and assume that $h(X)$ and $H(\lfloor X\rfloor)$ are finite. Suppose the sequence of almost-regular quantizers $q(\cdot)$ (parametrized by $D)$ satisfying the distortion constraint $\mathrm{E}\left[|X-q(X)|^{r}\right] \leq D$ achieves the asymptotic excess rate

$$
\frac{\lim }{D \downarrow 0}\{H(q(X))-R(D)\}=\frac{1}{r} \log \left(\frac{\Gamma(1+1 / r)^{r} e}{1+1 / r}\right) .
$$

Then,

$$
\varlimsup_{D \downarrow 0} \sum_{i} p_{i} \mathbf{1}\left\{\left|\frac{\Delta_{i}^{r}}{D}-V_{1}^{r}(1+r)\right| \leq \vartheta\right\}=1
$$

for every $\vartheta>0$.

Defining the function

$$
\Delta(x) \triangleq \sum_{i} \Delta_{i} \mathbf{1}\left\{x \in \mathcal{S}_{i}\right\}, \quad x \in \mathbb{R}
$$

the necessary condition (89) can be written as

$$
\varlimsup_{D \downarrow 0} \operatorname{Pr}\left(\left|\frac{\Delta^{r}(X)}{D}-V_{1}^{r}(1+r)\right| \leq \vartheta\right)=1
$$

for every $\vartheta>0$. Similar to Theorem 7 , Corollary 8 can thus be paraphrased as "any sequence of almost-regular quantizers achieves the asymptotic excess rate (88) only if $\Delta^{r}(X) / D$ converges in probability to $V_{1}^{r}(1+r)$ as $D \downarrow 0$." In other words, any sequence of almost-regular quantizers achieving $\mathrm{R}_{r, 1}$ must converge in probability to a uniform quantizer as $D$ tends to zero.

\section{Balls Versus Tessellating Polytopes}

The lower bound (22) on the asymptotic excess rate presented in Theorem 1 hinges on the fact that the distortion over the quantization region $\mathcal{S}_{i}$, i.e., $\int_{\mathcal{S}_{i}}\|\mathbf{x}-\hat{\mathbf{x}}\|^{r} \mathrm{dx}$, is lowerbounded by the distortion over a ball around $\hat{\mathbf{x}}_{i}$ with the same volume (cf. (101) in the proof of Lemma 6 with $\mathcal{B}_{i, \epsilon}$ replaced by $\mathcal{S}_{i}$ and with $\Lambda_{i, \epsilon}$ replaced by $\Delta_{i}$ ). Since the onedimensional ball is an interval and, hence, tessellates $\mathbb{R}$, it follows that for one-dimensional sources the lower bound (22) is achieved by a tessellating quantizer, so in this case it is tight. However, it is expected that this is no longer true for multidimensional sources, since in general balls do not tessellate the space. In fact, it is unclear whether there exists any (possibly non-tessellating) vector quantizer that achieves (22) for multidimensional sources.

To assess the tightness of the obtained lower bound, we compare it numerically with the asymptotic excess rates achievable by several lattice quantizers. To this end, we use Linder and Zeger's upper bound for tessellating quantizers (28) together with the normalized second moments $\ell(\mathcal{P})$ of various lattice quantizers tabulated in [42, Table I]. In order to better compare our results with previous works, in this section we consider the asymptotic excess rate per dimension, defined as $\overline{\mathrm{R}}_{r, d} \triangleq \mathrm{R}_{r, d} / d$. The asymptotic excess rate per dimension is relevant, for example, in the analysis of quantization schemes that buffer $d$ consecutive symbols of a one-dimensional source and then quantize them using a $d$-dimensional vector quantizer.
For the sake of simplicity, in this section we limit ourselves to the squared-error distortion $\mathbf{E}\left[\|\mathbf{X}-\hat{\mathbf{X}}\|_{2}^{2}\right] \leq D$, where

$$
\|\mathbf{a}\|_{2} \triangleq \sqrt{a_{1}^{2}+\ldots+a_{d}^{2}}, \quad \mathbf{a}=\left(a_{1}, \ldots, a_{d}\right) \in \mathbb{R}^{d}
$$

denotes the Euclidean norm. In this case, the lower bound (22) becomes

$$
\overline{\mathrm{R}}_{2, d} \geq \frac{1}{2} \log \left(2 \pi e \frac{\Gamma(1+d / 2)^{2 / d}}{\pi(2+d)}\right) .
$$

Furthermore, the upper bound corresponding to tessellating quantizers (28) becomes

$$
\overline{\mathrm{R}}_{2, d} \leq \frac{1}{2} \log \left(2 \pi e \frac{1}{d} \ell(\mathcal{P})\right) .
$$

It can be shown that the RHS of (94), when minimized over all $d$-dimensional, tessellating, convex polytopes $\mathcal{P}$, vanishes as $d$ tends to infinity. Indeed, [43, Lemma 1] (attributed to Poltyrev) demonstrates that $\frac{1}{d} \inf _{\mathcal{P}} \ell(\mathcal{P}) \rightarrow 1 /(2 \pi e)$ as $d$ tends to infinity. This is perhaps not very surprising, since the ratedistortion function $R(D)$ is essentially achieved by a vector quantizer whose dimension tends to infinity. By applying Zador's upper bound (13) on $b_{r, d}$ to (17), we further obtain

$$
\overline{\mathrm{R}}_{2, d} \leq \frac{1}{2} \log \left(2 \pi e \frac{\Gamma(1+2 / d) \Gamma(1+d / 2)^{2 / d}}{\pi d}\right) .
$$

Observe that the RHS of (95) also vanishes as $d$ tends to infinity. While this upper bound is in general looser than (94), it does not depend on the normalized second moment $\ell(\mathcal{P})$ of a tessellating quantizer and is therefore easier to evaluate.

In Figure 2, we depict the bounds (93) and (95) as a function of the dimension $d$. We further show several achievability results based on lattice quantizers (94). The normalized second moments $\ell(\mathcal{P})$ corresponding to these lattice quantizers were tabulated by Conway and Sloane in [42, Table I]. In fact, Figure 2 is equivalent to [42, Figure 1] with the only difference that here we plot the asymptotic excess rate per dimension whereas Conway and Sloane plot the normalized second moment. Specifically, we include the excess rates per dimension attained by a (one-dimensional) uniform quantizer, by a (two-dimensional) hexagonal quantizer, and by the three-dimensional tessellating quantizer whose regions are cuboctahedrons. These quantizers correspond to the socalled Voronoi lattices of the first type $A_{1}^{*}$ (the integers), $A_{2}^{*}$ (the two-dimensional hexagonal lattice), and $A_{3}^{*}$ (the bodycentered cubic lattice). For $d \geq 3$, we further include the excess rates per dimension attained by the $D_{d}^{*}$ lattices. Labeled with cross markers, we show the excess rates per dimension corresponding to the lattices $E_{6}^{*}, E_{7}^{*}$, the Gosset lattice $E_{8}$, the Coxeter-Todd lattice $K_{12}$, the Barnes-Wall lattice $\Lambda_{16}$, and the Leech lattice $\Lambda_{24}$. We refer to [42] and references therein for further details.

Finally, we compare the obtained bounds with a conjectured lower bound by Conway and Sloane [42, eq. (4)] that follows by computing the distortion attained by a set of reconstruction points located at the vertices of a $d$-dimensional tetrahedron. Note that this bound was computed for fixed-rate quantizers, i.e., for quantizers that have a fixed number $M$ of quantization regions and whose rate is defined as $\log M$. While the 


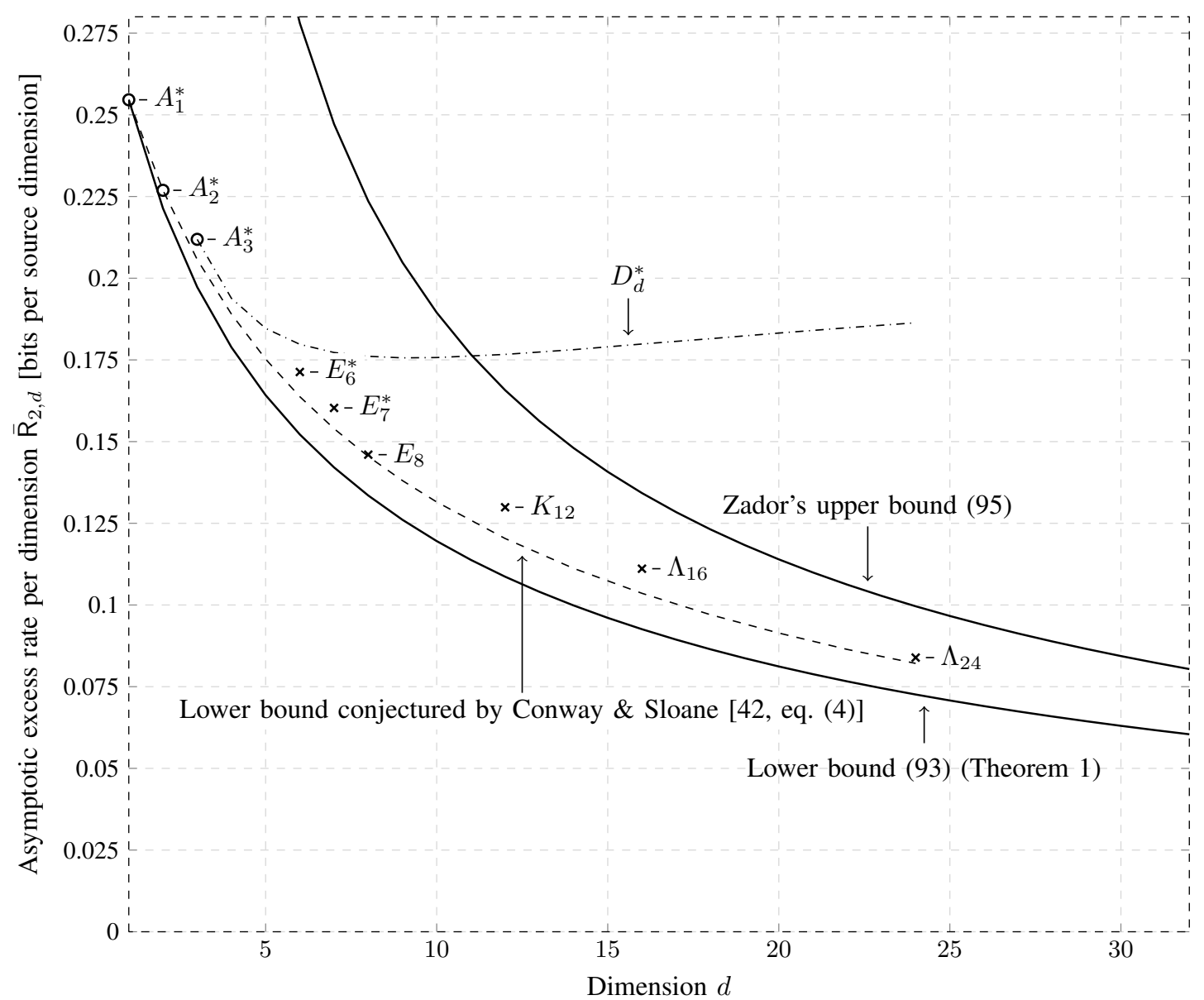

Figure 2. Bounds on the asymptotic excess rate per dimension $\overline{\mathrm{R}}_{2, d}$ (in bits per source dimension) of a $d$-dimensional vector quantizer. The excess rates per dimension attained by lattice quantizers were obtained by applying to (94) the normalized second moments tabulated in [42, Table I].

asymptotic excess rate achievable by a fixed-rate quantizer can also be achieved by an entropy-constrained quantizer, the converse is not necessarily true. It is thus prima facie unclear whether Conway and Sloane's conjectured lower bound would also apply to entropy-constrained quantizers. Nevertheless, we decided to include it here since it is remarkably close to the excess rates per dimension attained by the lattices $E_{8}$ and $\Lambda_{24}$.

As observed above, the asymptotic excess rate per dimension vanishes as $d$ tends to infinity. However, as illustrated by Figure 2, it decays slowly: for example, for a 10-dimensional vector quantizer we still have

$$
\begin{aligned}
\overline{\mathrm{R}}_{2,10} & \geq \frac{1}{2} \log _{2}\left(\frac{e \Gamma(6)^{1 / 5}}{6}\right) \\
& \approx 0.1196 \text { bits per source dimension }
\end{aligned}
$$

which is, arguably, not much smaller than the asymptotic excess rate per dimension of the (one-dimensional) uniform quantizer

$$
\overline{\mathrm{R}}_{2,1}=\frac{1}{2} \log _{2}(\pi e / 6) \approx 0.2546 \text { bits per source dimension. }
$$

(Here $\log _{2}(\cdot)$ denotes the binary logarithm.) In general, the bounds on $\overline{\mathrm{R}}_{2, d}$ given in (93) and (95) are of the order $\Theta(\log d / d)$.
Observe that for multi-dimensional sources the gap between the lower bound (93) and the asymptotic excess rate per dimension achievable with lattice quantizers is substantial. This gap is (at least partly) due to the fact that, in order to derive the lower bound (22), we lower-bounded the distortion over the quantization region $\mathcal{S}_{i}$ by that over a ball with the same volume. To obtain a tighter lower bound, one may need a more accurate approximation of this distortion that, like the conjectured bound by Conway and Sloane, takes the geometry of the optimal quantization regions into account.

\section{CONCLUSIONS}

The nonnegativity of relative entropy implies that the differential entropy of a random vector $\mathbf{X}$ with pdf $f$ is upperbounded by $-\mathrm{E}[\log g(\mathbf{X})]$ for any arbitrary pdf $g$. Using this inequality with a cleverly chosen $g$, we derived a lower bound on the asymptotic excess rate of entropy-constrained vector quantization. Specialized to the one-dimensional case and quadratic distortion, this bound coincides with the excess rate obtained by Gish and Pierce [6] and by Gray et al. [15]. The proposed derivation thus recovers the well-known result that uniform quantizers are asymptotically optimal as the allowed distortion vanishes. While the obtained lower bound itself is not novel, to the best of our knowledge, we presented the 
first rigorous derivation that follows the direct approach of Gish and Pierce without resorting to heuristic high-resolution approximations commonly found in the quantization literature.

Our result holds for any $d$-dimensional source $\mathbf{X}$ that satisfies $|h(\mathbf{X})|<\infty$ and $H(\lfloor\mathbf{X}\rfloor)<\infty$. The presented proof thus holds under the same conditions on the source as the proof by Gray et al., and it is more general than the proof by Gish and Pierce. In fact, it has recently been shown that these conditions are necessary and sufficient for the Shannon lower bound to be asymptotically tight for vanishing distortion, and that $H(\lfloor\mathbf{X}\rfloor)<\infty$ is a necessary and sufficient condition for the rate-distortion function to be finite [12]. Our result thus holds for the most general conditions that can be imposed in the analysis of high-resolution quantizers.

The derivation of the lower bound reveals a necessary condition for a sequence of quantizers (parametrized by $D$ ) to achieve the asymptotic excess rate. Specifically, we demonstrated for one-dimensional sources that the intersection of the quantization region $\mathcal{S}_{i}$ with the interval $\left[\hat{x}_{i}-\rho D^{1 / r}, \hat{x}_{i}+\right.$ $\rho D^{1 / r}$ must have a Lebesgue measure that converges in probability to $V_{1}(1+r)^{1 / r} D^{1 / r}$ as $D \rightarrow 0$ and $\rho \rightarrow \infty$. This implies that any sequence of almost-regular quantizers achieving the asymptotic excess rate must converge in probability to a uniform quantizer as $D \rightarrow 0$. Since almost-regular quantizers achieve $D_{r, 1}(R)$ when $r \geq 1$, this in turn suggests that asymptotically-optimal quantizers must essentially be uniform.

While the presented bound is tight for one-dimensional sources, it is unclear whether the same is true for multidimensional sources. Indeed, its derivation hinges on the fact that the distortion over the quantization region $\mathcal{S}_{i}$ is lowerbounded by the distortion over a ball around $\hat{\mathbf{x}}_{i}$ with the same volume. Since the one-dimensional ball is an interval and, hence, tessellates $\mathbb{R}$, it follows that for one-dimensional sources the converse bound (22) is achieved by a tessellating quantizer (which in this case is the uniform quantizer). However, it is expected that this is no longer true for multidimensional sources, since in general balls do not tessellate the space. It is yet unclear whether there exists any (possibly non-tessellating) vector quantizer that achieves our converse bound for multi-dimensional sources.

\section{APPENDIX A}

\section{PROOF OF LEMMA 6}

To prove Lemma 6 , we first fix an arbitrary constant $\eta>0$ and divide the indices $i$ according to whether $\Lambda_{i, \epsilon} \geq \eta V_{d} \epsilon^{d}$ or not. Specifically, let

$$
\mathcal{I} \triangleq\left\{i \in \mathbb{Z}: \Lambda_{i, \epsilon} \geq \eta V_{d} \epsilon^{d}\right\}
$$

and divide the sum on the LHS of (74) into

$$
\sum_{i} p_{i, \epsilon} \frac{\Lambda_{i, \epsilon}^{r / d}}{D}=\sum_{i \in \mathcal{I}} p_{i, \epsilon} \frac{\Lambda_{i, \epsilon}^{r / d}}{D}+\sum_{i \in \mathcal{I}^{\mathrm{c}}} p_{i, \epsilon} \frac{\Lambda_{i, \epsilon}^{r / d}}{D}
$$

where $\mathcal{I}^{\mathrm{c}}$ denotes the complement of $\mathcal{I}$. For every $i \in \mathcal{I}^{\mathrm{c}}$ we have $\Lambda_{i, \epsilon}<\eta V_{d} \epsilon^{d}$, so the second sum on the RHS of (98) can be upper-bounded as

$$
\sum_{i \in \mathcal{I}^{\mathrm{c}}} p_{i, \epsilon} \frac{\Lambda_{i, \epsilon}^{r / d}}{D} \leq \eta^{r / d} V_{d}^{r / d} \frac{\epsilon^{r}}{D} \sum_{i \in \mathcal{I}^{\mathrm{c}}} p_{i, \epsilon} \leq \eta^{r / d} \frac{V_{d}^{r / d}}{\kappa}
$$

where the second step follows because $\epsilon^{r}=D / \kappa$ and because, by definition, the sets $\mathcal{B}_{i, \epsilon}$ are disjoint, so the sum of the probabilities $p_{i, \epsilon}$ is equal to the probability of $\cup_{i \in \mathcal{I}^{\mathrm{c}}} \mathcal{B}_{i, \epsilon}$, which is upper-bounded by 1 .

To upper-bound the first sum on the RHS of (98), we begin by lower-bounding $\mathrm{E}\left[\|\mathbf{X}-\hat{\mathbf{X}}\|^{r}\right]$ as

$$
\begin{aligned}
\mathrm{E}[ & \left.\|\mathbf{X}-\hat{\mathbf{X}}\|^{r}\right] \\
= & \sum_{i} \int_{\mathcal{S}_{i}} f_{\mathbf{X}}(\mathbf{x})\left\|\mathbf{x}-\hat{\mathbf{x}}_{i}\right\|^{r} \mathrm{~d} \mathbf{x} \\
\geq & \sum_{i \in \mathcal{I}} \int_{\mathcal{B}_{i, \epsilon}} f_{\mathbf{X}}(\mathbf{x})\left\|\mathbf{x}-\hat{\mathbf{x}}_{i}\right\|^{r} \mathrm{~d} \mathbf{x} \\
= & \sum_{i \in \mathcal{I}} \frac{p_{i, \epsilon}}{\Lambda_{i, \epsilon}} \int_{\mathcal{B}_{i, \epsilon}}\left\|\mathbf{x}-\hat{\mathbf{x}}_{i}\right\|^{r} \mathrm{~d} \mathbf{x} \\
& -\sum_{i \in \mathcal{I}} \int_{\mathcal{B}_{i, \epsilon}}\left[\frac{p_{i, \epsilon}}{\Lambda_{i, \epsilon}}-f_{\mathbf{X}}(\mathbf{x})\right]\left\|\mathbf{x}-\hat{\mathbf{x}}_{i}\right\|^{r} \mathrm{~d} \mathbf{x} .
\end{aligned}
$$

The region $\mathcal{B}_{i, \epsilon}$ of volume $\Lambda_{i, \epsilon}$ that minimizes the integral $\int_{\mathcal{B}_{i, \epsilon}}\|\mathbf{x}-\hat{\mathbf{x}}\|^{r} \mathrm{~d} \mathbf{x}$ is a ball around $\hat{\mathbf{x}}$. We thus have [8, Section III]

$$
\frac{1}{\Lambda_{i, \epsilon}} \int_{\mathcal{B}_{i, \epsilon}}\left\|\mathbf{x}-\hat{\mathbf{x}}_{i}\right\|^{r} \mathrm{~d} \mathbf{x} \geq \frac{d}{d+r} \frac{\Lambda_{i, \epsilon}^{r / d}}{V_{d}^{r / d}}
$$

which yields for the first term on the RHS of (100)

$$
\sum_{i \in \mathcal{I}} \frac{p_{i, \epsilon}}{\Lambda_{i, \epsilon}} \int_{\mathcal{B}_{i, \epsilon}}\left\|\mathbf{x}-\hat{\mathbf{x}}_{i}\right\|^{r} \mathrm{~d} \mathbf{x} \geq \sum_{i \in \mathcal{I}} p_{i, \epsilon} \frac{\Lambda_{i, \epsilon}^{r / d}}{V_{d}^{r / d}(1+r / d)}
$$

Multiplying both sides of (100) by $V_{d}^{r / d}(1+r / d) / D$, applying (102) to (100), and using that $\mathrm{E}\left[\|\mathbf{X}-\hat{\mathbf{X}}\|^{r}\right] \leq D$, we obtain

$$
\begin{aligned}
& \sum_{i \in \mathcal{I}} p_{i, \epsilon} \frac{\Lambda_{i, \epsilon}^{r / d}}{D} \leq V_{d}^{r / d}\left(1+\frac{r}{d}\right) \\
& +\frac{V_{d}^{r / d}\left(1+\frac{r}{d}\right)}{D} \sum_{i \in \mathcal{I}} \int_{\mathcal{B}_{i, \epsilon}}\left[\frac{p_{i, \epsilon}}{\Lambda_{i, \epsilon}}-f_{\mathbf{X}}(\mathbf{x})\right]\left\|\mathbf{x}-\hat{\mathbf{x}}_{i}\right\|^{r} \mathrm{~d} \mathbf{x} .
\end{aligned}
$$

We next introduce the pdf

$$
\begin{aligned}
& f_{\mathbf{X}}^{(\Lambda)}\left(\mathbf{x} ;\left\{\mathcal{B}_{i, \epsilon}\right\}\right) \triangleq \sum_{i \in \mathcal{I}} \frac{p_{i, \epsilon}}{\Lambda_{i, \epsilon}} \mathbf{1}\left\{\mathbf{x} \in \mathcal{B}_{i, \epsilon}\right\} \\
& \quad+f_{\mathbf{X}}(\mathbf{x})\left[\sum_{i \in \mathcal{I}} \mathbf{1}\left\{\mathbf{x} \in \overline{\mathcal{B}}_{i, \epsilon}\right\}+\sum_{i \in \mathcal{I}^{c}} \mathbf{1}\left\{\mathbf{x} \in \mathcal{S}_{i}\right\}\right]
\end{aligned}
$$

for $\mathbf{x} \in \mathbb{R}^{d}$, which allows us to write

$$
\begin{aligned}
& \sum_{i \in \mathcal{I}} \int_{\mathcal{B}_{i, \epsilon}}\left[\frac{p_{i, \epsilon}}{\Lambda_{i, \epsilon}}-f_{\mathbf{X}}(\mathbf{x})\right]\left\|\mathbf{x}-\hat{\mathbf{x}}_{i}\right\|^{r} \mathrm{~d} \mathbf{x} \\
& =\sum_{i} \int_{\mathcal{S}_{i}}\left[f_{\mathbf{X}}^{(\Lambda)}\left(\mathbf{x} ;\left\{\mathcal{B}_{i, \epsilon}\right\}\right)-f_{\mathbf{X}}(\mathbf{x})\right]\left\|\mathbf{x}-\hat{\mathbf{x}}_{i}\right\|^{r} \mathrm{~d} \mathbf{x} .
\end{aligned}
$$


Since $\left\|\mathbf{x}-\hat{\mathbf{x}}_{i}\right\|^{r} \leq \epsilon^{r}=D / \kappa$ for $\mathbf{x} \in \mathcal{B}_{i, \epsilon}, i \in \mathcal{I}$ and $f_{\mathbf{X}}^{(\Lambda)}\left(\mathbf{x} ;\left\{\mathcal{B}_{i, \epsilon}\right\}\right)-f_{\mathbf{X}}(\mathbf{x})=0$ otherwise, we have

$$
\begin{array}{r}
\left|\sum_{i} \int_{\mathcal{S}_{i}}\left[f_{\mathbf{X}}^{(\Lambda)}\left(\mathbf{x} ;\left\{\mathcal{B}_{i, \epsilon}\right\}\right)-f_{\mathbf{X}}(\mathbf{x})\right]\left\|\mathbf{x}-\hat{\mathbf{x}}_{i}\right\|^{r} \mathrm{~d} \mathbf{x}\right| \\
\leq \frac{D}{\kappa} \int\left|f_{\mathbf{X}}^{(\Lambda)}\left(\mathbf{x} ;\left\{\mathcal{B}_{i, \epsilon}\right\}\right)-f_{\mathbf{X}}(\mathbf{x})\right| \mathrm{d} \mathbf{x} .
\end{array}
$$

Combining this upper bound with (98), (99), and (103), we obtain

$$
\begin{aligned}
& \sum_{i} p_{i, \epsilon} \frac{\Lambda_{i, \epsilon}^{r / d}}{D} \leq \eta^{r / d} \frac{V_{d}^{r / d}}{\kappa}+V_{d}^{r / d}\left(1+\frac{r}{d}\right) \\
& +\frac{V_{d}^{r / d}\left(1+\frac{r}{d}\right)}{\kappa} \int\left|f_{\mathbf{X}}^{(\Lambda)}\left(\mathbf{x} ;\left\{\mathcal{B}_{i, \epsilon}\right\}\right)-f_{\mathbf{X}}(\mathbf{x})\right| \mathrm{d} \mathbf{x} .
\end{aligned}
$$

We next show that, for every $\eta>0$,

$$
\lim _{D \downarrow 0} \sup _{q(\cdot)} \int\left|f_{\mathbf{X}}^{(\Lambda)}\left(\mathbf{x} ;\left\{\mathcal{B}_{i, \epsilon}\right\}\right)-f_{\mathbf{X}}(\mathbf{x})\right| \mathrm{d} \mathbf{x}=0 .
$$

(Recall that $f_{\mathbf{X}}^{(\Lambda)}$ depends on $q(\cdot)$ and $D$ via $\left\{\mathcal{B}_{i, \epsilon}\right\}$ and on $\eta$ via $\mathcal{I}$.) It then follows that

$$
\varlimsup_{D \downarrow 0} \sup _{q(\cdot)} \sum_{i} p_{i, \epsilon} \frac{\Lambda_{i, \epsilon}^{r / d}}{D} \leq V_{d}^{r / d}\left(1+\frac{r}{d}\right)+\eta^{r / d} \frac{V_{d}^{r / d}}{\kappa}
$$

which proves Lemma 6 upon letting $\eta$ tend to zero from above.

It thus remains to prove (108). By definition, $f_{\mathbf{X}}^{(\Lambda)}$ differs from $f_{\mathbf{X}}$ only when $\mathbf{x} \in \mathcal{B}_{i, \epsilon}, i \in \mathcal{I}$. Since the families of sets $\mathcal{B}_{i, \epsilon}, i \in \mathcal{I}$ (parametrized by $D$ ) have bounded eccentricity, it follows from Lebesgue's differentiation theorem that $f_{\mathbf{X}}^{(\Lambda)}$ converges to $f_{\mathbf{X}}$ almost everywhere as $D$ (and hence also $\epsilon$ ) tends to zero. By Scheffe's lemma, this then implies (108). However, compared to the standard setting under which Lebesgue's differentiation theorem is proven, our setting is slightly more complicated, since as $D$ tends to zero not only the diameters of the sets $\mathcal{B}_{i, \epsilon}$ decay, but also their locations in $\mathbb{R}^{d}$ may change. For completeness, we therefore provide all the steps, even though they follow closely the standard proof of the Lebesgue differentiation theorem.

We first note that the integral in (108) is nonnegative and bounded, so its supremum is finite and for every $\nu>0$ there exists a sequence of quantizers (parametrized by $D$ ) such that

$$
\begin{aligned}
& \varlimsup_{D \downarrow 0} \int\left|f_{\mathbf{X}}^{(\Lambda)}\left(\mathbf{x} ;\left\{\mathcal{B}_{i, \epsilon}\right\}\right)-f_{\mathbf{X}}(\mathbf{x})\right| \mathrm{d} \mathbf{x} \\
& \quad \geq \varlimsup_{D \downarrow 0} \sup _{q(\cdot)} \int\left|f_{\mathbf{X}}^{(\Lambda)}\left(\mathbf{x} ;\left\{\mathcal{B}_{i, \epsilon}\right\}\right)-f_{\mathbf{X}}(\mathbf{x})\right| \mathrm{d} \mathbf{x}-\nu .
\end{aligned}
$$

Since $\nu>0$ is arbitrary, in order to prove (108) it suffices to show that for any sequence of quantizers (parametrized by $D$ )

$$
\lim _{D \downarrow 0} \int\left|f_{\mathbf{X}}^{(\Lambda)}\left(\mathbf{x} ;\left\{\mathcal{B}_{i, \epsilon}\right\}\right)-f_{\mathbf{X}}(\mathbf{x})\right| \mathrm{d} \mathbf{x}=0 .
$$

Specifically, we shall show that for any sequence of quantizers (parametrized by $D$ )

$$
\lambda\left(\left\{\mathbf{x} \in \mathbb{R}^{d}: \varlimsup_{D \downarrow 0}\left|f_{\mathbf{X}}^{(\Lambda)}\left(\mathbf{x} ;\left\{\mathcal{B}_{i, \epsilon}\right\}\right)-f_{\mathbf{X}}(\mathbf{x})\right|>2 \xi\right\}\right)=0
$$

for every $\xi>0$, where $\lambda(\cdot)$ denotes the Lebesgue measure on $\mathbb{R}^{d}$. It then follows that $f_{\mathbf{X}}^{(\Lambda)}$ converges to $f_{\mathbf{X}}$ almost everywhere as $D \rightarrow 0$ since

$$
\begin{aligned}
& \left\{\mathbf{x} \in \mathbb{R}^{d}: \varlimsup_{D \downarrow 0}\left|f_{\mathbf{X}}^{(\Lambda)}\left(\mathbf{x} ;\left\{\mathcal{B}_{i, \epsilon}\right\}\right)-f_{\mathbf{X}}(\mathbf{x})\right|>0\right\} \\
& =\bigcup_{\ell=1}^{\infty}\left\{\mathbf{x} \in \mathbb{R}^{d}: \varlimsup_{D \downarrow 0}\left|f_{\mathbf{X}}^{(\Lambda)}\left(\mathbf{x} ;\left\{\mathcal{B}_{i, \epsilon}\right\}\right)-f_{\mathbf{X}}(\mathbf{x})\right|>\frac{1}{\ell}\right\}
\end{aligned}
$$

and the countable union of sets of measure zero has measure zero. By Scheffe's lemma, almost everywhere convergence of $f_{\mathbf{X}}^{(\Lambda)}$ to $f_{\mathbf{X}}$ implies (111), which together with (110) proves the desired result (108) upon letting $\nu$ tend to zero from above.

We thus set out to prove (112). By the definition of $f_{\mathbf{X}}^{(\Lambda)}$ and the triangle inequality,

$$
\begin{aligned}
\mid f_{\mathbf{X}}^{(\Lambda)}\left(\mathbf{x} ;\left\{\mathcal{B}_{i, \epsilon}\right\}\right) & -f_{\mathbf{X}}(\mathbf{x}) \mid \\
& \leq \sum_{i \in \mathcal{I}}\left|\frac{p_{i, \epsilon}}{\Lambda_{i, \epsilon}}-f_{\mathbf{X}}(\mathbf{x})\right| \mathbf{1}\left\{\mathbf{x} \in \mathcal{B}_{i, \epsilon}\right\} .
\end{aligned}
$$

We next approximate $p_{i, \epsilon}$ by replacing $f_{\mathbf{X}}$ by a continuous function $g$. Indeed, since $f_{\mathbf{X}}$ is integrable, for every $\varepsilon>0$ there exists a continuous function $g$ such that [44, Theorem 2.4.14, p. 92]

$$
\int\left|f_{\mathbf{X}}(\mathbf{x})-g(\mathbf{x})\right| \mathrm{d} \mathbf{x} \leq \varepsilon .
$$

It then follows that, for every $\mathrm{x} \in \mathcal{B}_{i, \epsilon}$,

$$
\begin{aligned}
& \left|\frac{p_{i, \epsilon}}{\Lambda_{i, \epsilon}}-f_{\mathbf{X}}(\mathbf{x})\right| \leq\left|\frac{1}{\Lambda_{i, \epsilon}} \int_{\mathcal{B}_{i, \epsilon}} g(\mathbf{y}) \mathrm{d} \mathbf{y}-g(\mathbf{x})\right| \\
& \quad+\frac{1}{\Lambda_{i, \epsilon}} \int_{\mathcal{B}_{i, \epsilon}}\left|f_{\mathbf{X}}(\mathbf{y})-g(\mathbf{y})\right| \mathrm{d} \mathbf{y}+\left|f_{\mathbf{X}}(\mathbf{x})-g(\mathbf{x})\right| .
\end{aligned}
$$

Let $\mathcal{B}(\mathbf{c}, \rho) \triangleq\left\{\mathbf{x} \in \mathbb{R}^{d}:\|\mathbf{x}-\mathbf{c}\| \leq \rho\right\}$ denote the $d$-dimensional ball of radius $\rho$ centered at $\mathbf{c}$. Note that $\lambda\left(\mathcal{B}\left(\hat{\mathbf{x}}_{i}, \epsilon\right)\right)=V_{d} \epsilon^{d}$. For every $\mathbf{x} \in \mathcal{B}_{i, \epsilon}$ and $i \in \mathcal{I}$, the second term on the RHS of (116) can be upper-bounded by

$$
\begin{aligned}
& \frac{1}{\Lambda_{i, \epsilon}} \int_{\mathcal{B}_{i, \epsilon}}\left|f_{\mathbf{X}}(\mathbf{y})-g(\mathbf{y})\right| \mathrm{d} \mathbf{y} \\
& \quad \leq \frac{1}{\eta \lambda\left(\mathcal{B}\left(\hat{\mathbf{x}}_{i}, \epsilon\right)\right)} \int_{\mathcal{B}\left(\hat{\mathbf{x}}_{i}, \epsilon\right)}\left|f_{\mathbf{X}}(\mathbf{y})-g(\mathbf{y})\right| \mathrm{d} \mathbf{y} \\
& \quad \leq \frac{2^{d}}{\eta} \frac{1}{\lambda(\mathcal{B}(\mathbf{x}, 2 \epsilon))} \int_{\mathcal{B}(\mathbf{x}, 2 \epsilon)}\left|f_{\mathbf{X}}(\mathbf{y})-g(\mathbf{y})\right| \mathrm{d} \mathbf{y} \\
& \quad \leq \frac{2^{d}}{\eta}\left(f_{\mathbf{X}}-g\right)^{\star}(\mathbf{x})
\end{aligned}
$$

where $\left(f_{\mathbf{X}}-g\right)^{\star}$ denotes the Hardy-Littlewood maximal function for $f_{\mathbf{X}}-g$, i.e.,

$$
\left(f_{\mathbf{X}}-g\right)^{\star}(\mathbf{x}) \triangleq \sup _{\rho>0} \frac{1}{\lambda(\mathcal{B}(\mathbf{x}, \rho))} \int_{\mathcal{B}(\mathbf{x}, \rho)}\left|f_{\mathbf{X}}(\mathbf{y})-g(\mathbf{y})\right| \mathrm{d} \mathbf{y}
$$

for $\mathrm{x} \in \mathbb{R}^{d}$. In (117) we have used that, for every $\mathrm{x} \in \mathcal{B}_{i, \epsilon}$ and $i \in \mathcal{I}$, we have $\mathcal{B}_{i, \epsilon} \subseteq \mathcal{B}\left(\hat{\mathbf{x}}_{i}, \epsilon\right) \subseteq \mathcal{B}(\mathbf{x}, 2 \epsilon)$ and, by the definition of $\mathcal{I}$,

$$
\Lambda_{i, \epsilon} \geq \eta \lambda\left(\mathcal{B}\left(\hat{\mathbf{x}}_{i}, \epsilon\right)\right)=2^{-d} \lambda(\mathcal{B}(\mathbf{x}, 2 \epsilon)) .
$$


Combining (116) and (117) with (114), we obtain

$$
\begin{aligned}
& \left|f_{\mathbf{X}}^{(\Lambda)}\left(\mathbf{x} ;\left\{\mathcal{B}_{i, \epsilon}\right\}\right)-f_{\mathbf{X}}(\mathbf{x})\right| \\
& \leq \sum_{i \in \mathcal{I}}\left|\frac{1}{\Lambda_{i, \epsilon}} \int_{\mathcal{B}_{i, \epsilon}} g(\mathbf{y}) \mathrm{d} \mathbf{y}-g(\mathbf{x})\right| \mathbf{1}\left\{\mathbf{x} \in \mathcal{B}_{i, \epsilon}\right\} \\
& \quad+\sum_{i \in \mathcal{I}} \frac{2^{d}}{\eta}\left(f_{\mathbf{X}}-g\right)^{\star}(\mathbf{x}) \mathbf{1}\left\{\mathbf{x} \in \mathcal{B}_{i, \epsilon}\right\} \\
& \quad+\sum_{i \in \mathcal{I}}\left|f_{\mathbf{X}}(\mathbf{x})-g(\mathbf{x})\right| \mathbf{1}\left\{\mathbf{x} \in \mathcal{B}_{i, \epsilon}\right\} \\
& \leq \sum_{i \in \mathcal{I}}\left|\frac{1}{\Lambda_{i, \epsilon}} \int_{\mathcal{B}_{i, \epsilon}} g(\mathbf{y}) \mathrm{d} \mathbf{y}-g(\mathbf{x})\right| \mathbf{1}\left\{\mathbf{x} \in \mathcal{B}_{i, \epsilon}\right\} \\
& \quad+\frac{2^{d}}{\eta}\left(f_{\mathbf{X}}-g\right)^{\star}(\mathbf{x})+\left|f_{\mathbf{X}}(\mathbf{x})-g(\mathbf{x})\right|, \quad \mathbf{x} \in \mathbb{R}^{d}
\end{aligned}
$$

where the second inequality follows because the sets $\left\{\mathcal{B}_{i, \epsilon}\right\}$ are disjoint. The second and third term on the RHS of (119) are independent of $D$ and $q(\cdot)$. The first term on the RHS of (119) vanishes as $D$ tends to zero for any sequence of quantizers. Indeed, the continuity of $g$ implies that for every $\mathbf{x} \in \mathbb{R}^{d}$ and $\vartheta>0$ there exists an $\epsilon_{0}>0$ such that

$$
|g(\mathbf{y})-g(\mathbf{x})| \leq \vartheta, \quad \text { for }\|\mathbf{x}-\mathbf{y}\| \leq 2 \epsilon_{0} .
$$

Since $\mathbf{x}, \mathbf{y} \in \mathcal{B}_{i, \epsilon}$ satisfy $\|\mathbf{x}-\mathbf{y}\| \leq 2 \epsilon$, it follows that for every $\mathbf{x} \in \mathbb{R}^{d}$ and $\vartheta>0$ there exists an $\epsilon_{0}>0$ such that

$$
\left|\frac{1}{\Lambda_{i, \epsilon}} \int_{\mathcal{B}_{i, \epsilon}} g(\mathbf{y}) \mathrm{d} \mathbf{y}-g(\mathbf{x})\right| \mathbf{1}\left\{\mathbf{x} \in \mathcal{B}_{i, \epsilon}\right\} \leq \vartheta \mathbf{1}\left\{\mathbf{x} \in \mathcal{B}_{i, \epsilon}\right\}
$$

for $\epsilon \leq \epsilon_{0}$. Using that the sets $\mathcal{B}_{i, \epsilon}, i \in \mathcal{I}$ are disjoint, we conclude that for every $\mathbf{x} \in \mathbb{R}^{d}$ and $\vartheta>0$ there exists an $\epsilon_{0}>0$ such that

$$
\sum_{i \in \mathcal{I}}\left|\frac{1}{\Lambda_{i, \epsilon}} \int_{\mathcal{B}_{i, \epsilon}} g(\mathbf{y}) \mathrm{d} \mathbf{y}-g(\mathbf{x})\right| \mathbf{1}\left\{\mathbf{x} \in \mathcal{B}_{i, \epsilon}\right\} \leq \vartheta
$$

for $\epsilon \leq \epsilon_{0}$. Since $\vartheta>0$ is arbitrary and $\epsilon$ vanishes as $D \rightarrow$ 0 , this implies that for every $\mathbf{x} \in \mathbb{R}^{d}$ and any sequence of quantizers

$$
\lim _{D \downarrow 0} \sum_{i \in \mathcal{I}}\left|\frac{1}{\Lambda_{i, \epsilon}} \int_{\mathcal{B}_{i, \epsilon}} g(\mathbf{y}) \mathrm{d} \mathbf{y}-g(\mathbf{x})\right| \mathbf{1}\left\{\mathbf{x} \in \mathcal{B}_{i, \epsilon}\right\}=0 .
$$

We conclude the proof of Lemma 6 by applying (119) and (123) to upper-bound the Lebesgue measure on the LHS of (112). Indeed, we have

$$
\begin{aligned}
& \lambda\left(\left\{\mathbf{x} \in \mathbb{R}^{d}: \varlimsup_{D \downarrow 0}\left|f_{\mathbf{X}}^{(\Lambda)}\left(\mathbf{x} ;\left\{\mathcal{B}_{i, \epsilon}\right\}\right)-f_{\mathbf{X}}(\mathbf{x})\right|>2 \xi\right\}\right) \\
& \leq \lambda\left(\left\{\mathbf{x} \in \mathbb{R}^{d}: \frac{2^{d}}{\eta}\left(f_{\mathbf{X}}-g\right)^{\star}(\mathbf{x})+\left|f_{\mathbf{X}}(\mathbf{x})-g(\mathbf{x})\right|>2 \xi\right\}\right) \\
& \leq \lambda\left(\left\{\mathbf{x} \in \mathbb{R}^{d}: \frac{2^{d}}{\eta}\left(f_{\mathbf{X}}-g\right)^{\star}(\mathbf{x})>\xi\right\}\right) \\
& \quad+\lambda\left(\left\{\mathbf{x} \in \mathbb{R}^{d}:\left|f_{\mathbf{X}}(\mathbf{x})-g(\mathbf{x})\right|>\xi\right\}\right) .
\end{aligned}
$$

The first term on the RHS of (124) can be upper-bounded by using the Hardy-Littlewood maximal inequality [45, Theorem 3.4, p. 55]

$$
\begin{aligned}
\lambda\left(\left\{\mathbf{x} \in \mathbb{R}^{d}: \frac{2^{d}}{\eta}\left(f_{\mathbf{X}}\right.\right.\right. & \left.\left.-g)^{\star}(\mathbf{x})>\xi\right\}\right) \\
& \leq \frac{2^{d} \alpha_{d}}{\eta \xi} \int\left|f_{\mathbf{X}}(\mathbf{x})-g(\mathbf{x})\right| \mathrm{d} \mathbf{x}
\end{aligned}
$$

for some constant $\alpha_{d}$ that only depends on $d$. Likewise, the second term on the RHS of (124) can be upper-bounded using Chebyshev's inequality [44, Theorem 4.10.7, p. 192]

$$
\begin{aligned}
\lambda\left(\left\{\mathbf{x} \in \mathbb{R}^{d}:\left|f_{\mathbf{X}}(\mathbf{x})-g(\mathbf{x})\right|>\xi\right\}\right) & \\
& \leq \frac{1}{\xi} \int\left|f_{\mathbf{X}}(\mathbf{x})-g(\mathbf{x})\right| \mathrm{d} \mathbf{x} .
\end{aligned}
$$

Combining (125) and (126) with (115) and (124), it follows that

$$
\begin{aligned}
\lambda\left(\left\{\mathbf{x} \in \mathbb{R}^{d}: \varlimsup_{D \downarrow 0} \mid f_{\mathbf{X}}^{(\Lambda)}\left(\mathbf{x} ;\left\{\mathcal{B}_{i, \epsilon}\right\}\right)\right.\right. & \left.\left.-f_{\mathbf{X}}(\mathbf{x}) \mid>2 \xi\right\}\right) \\
& \leq \frac{1+2^{d} \alpha_{d} / \eta}{\xi} \varepsilon .
\end{aligned}
$$

This proves (112) upon letting $\varepsilon$ tend to zero from above, which was the last step required to prove Lemma 6 .

\section{APPENDIX B}

PROOF OF THEOREM 7

Following the steps (60)-(70) in the proof of Theorem 1 specialized to the case $d=1$, we obtain that

$$
\begin{aligned}
& H(q(X))-R(D) \\
& \geq \frac{1}{r} \log \left(r V_{1}^{r} \Gamma(1+1 / r)^{r} e\right) \\
& \quad-\frac{1}{r} \sum_{i} p_{i, \epsilon} \log \left(\frac{\Lambda_{i, \epsilon}}{D^{1 / r}}+V_{1} \Gamma\left(\frac{1}{r}, \frac{1}{\kappa \delta}\right)\right)^{r} \\
& \quad-\kappa \log \left(\frac{V_{1}}{\kappa^{1 / r}}+V_{1} \Gamma(1 / r)\right)-\kappa\left|\log \frac{r}{\delta^{1 / r}}\right|-\frac{1}{\delta} .
\end{aligned}
$$

Recall that $\epsilon^{r}=D / \kappa$. The last three terms on the RHS of (128) are independent of $D$ and vanish as we first let $\kappa \rightarrow 0$ and then $\delta \rightarrow \infty$. To achieve

$$
\mathrm{R}_{r, 1}=\frac{1}{r} \log \left(\frac{\Gamma(1+1 / r)^{r} e}{1+1 / r}\right)
$$

a sequence of quantizers (parametrized by $D$ ) must therefore satisfy

$$
\begin{aligned}
\varliminf_{\kappa \downarrow 0} \varlimsup_{D \downarrow 0} \frac{1}{r} \sum_{i} p_{i, \epsilon} \log \left(\frac{\Lambda_{i, \epsilon}}{D^{1 / r}}\right. & \left.+V_{1} \Gamma\left(\frac{1}{r}, \frac{1}{\kappa \delta}\right)\right)^{r} \\
& \geq \frac{1}{r} \log \left(V_{1}^{r}(1+r)\right) .
\end{aligned}
$$

(As $\kappa \rightarrow 0$, the term on the LHS of (129) becomes independent of $\delta$.) For the sake of compactness, we shall use in the rest of the proof the following notation: ${ }^{5}$

\footnotetext{
${ }^{5}$ While some of the introduced quantities depend on $\kappa$ and $D$, to keep the notation compact we only make the dependence on $D$ explicit.
} 
Let $V \triangleq V_{1}^{r}(1+r)$. Further let $v \triangleq V_{1} \Gamma\left(\frac{1}{r}, \frac{1}{\kappa \delta}\right)$, and recall that $\lim _{\kappa \rightarrow 0} v=0$ for every $\delta>0$. Define

$$
\begin{aligned}
& \underline{\mathcal{I}}_{D} \triangleq\left\{i \in \mathbb{Z}: \frac{\Lambda_{i, \epsilon}^{r}}{D} \leq V-\vartheta\right\} \\
& \overline{\mathcal{I}}_{D} \triangleq\left\{i \in \mathbb{Z}: \frac{\Lambda_{i, \epsilon}^{r}}{D} \geq V+\vartheta\right\}
\end{aligned}
$$

for some $\vartheta>0$, and let

$$
\begin{aligned}
& \underline{q}_{D} \triangleq \frac{1}{1-\wp_{\epsilon}} \sum_{i \in \underline{\mathcal{I}}_{D}} p_{i, \epsilon} \\
& \bar{q}_{D} \triangleq \frac{1}{1-\wp_{\epsilon}} \sum_{i \in \overline{\mathcal{I}}_{D}} p_{i, \epsilon} \\
& \underline{\bar{q}}_{D} \triangleq \frac{1}{1-\wp_{\epsilon}} \sum_{i \in \mathbb{Z} \backslash\left(\underline{\mathcal{I}}_{D} \cup \overline{\mathcal{I}}_{D}\right)} p_{i, \epsilon}
\end{aligned}
$$

and

$$
\begin{aligned}
& \underline{\mu}_{D} \triangleq \frac{1}{\left(1-\wp_{\epsilon}\right) \underline{q}_{D}} \sum_{i \in \underline{\mathcal{I}}_{D}} p_{i, \epsilon} \frac{\Lambda_{i, \epsilon}^{r}}{D} \\
& \bar{\mu}_{D} \triangleq \frac{1}{\left(1-\wp_{\epsilon}\right) \bar{q}_{D}} \sum_{i \in \overline{\mathcal{I}}_{D}} p_{i, \epsilon} \frac{\Lambda_{i, \epsilon}^{r}}{D} \\
& \underline{\mu}_{D} \triangleq \frac{1}{\left(1-\wp_{\epsilon}\right) \underline{\bar{q}}_{D}} \sum_{i \in \mathbb{Z} \backslash\left(\underline{\mathcal{I}}_{D} \cup \overline{\mathcal{I}}_{D}\right)} p_{i, \epsilon} \frac{\Lambda_{i, \epsilon}^{r}}{D}
\end{aligned}
$$

where $\wp_{\epsilon}$ was defined in (67). Finally, define

$$
\mu_{D} \triangleq \underline{q}_{D} \underline{\mu}_{D}+\bar{q}_{D} \bar{\mu}_{D}+\underline{\bar{q}}_{D} \underline{\bar{\mu}}_{D} .
$$

By definition of $\underline{\mathcal{I}}$ and $\overline{\mathcal{I}}$, we have

$$
\underline{\mu}_{D} \leq V-\vartheta \quad \text { and } \quad \bar{\mu}_{D} \geq V+\vartheta .
$$

Furthermore, by Lemma 6 and (68),

$$
\varlimsup_{\kappa \downarrow 0} \varlimsup_{D \downarrow 0} \mu_{D} \leq V .
$$

Consequently, for any arbitrary $\varepsilon>0$, there exist $\kappa_{0}$ and $D_{0}$ such that

$$
\mu_{D} \leq V+\varepsilon, \quad\left(\kappa \leq \kappa_{0}, D \leq D_{0}\right) .
$$

Without loss of generality, we implicitly assume that $\kappa$ and $D$ are sufficiently small, so that (136) holds.

We next apply steps similar to (71) and (72) to upper-bound

$$
\begin{array}{r}
\sum_{i} \frac{p_{i, \epsilon}}{1-\wp_{\epsilon}} \log \left(\frac{\Lambda_{i, \epsilon}}{D^{1 / r}}+v\right)^{r} \leq \underline{q}_{D} \log \left(\underline{\mu}_{D}+v^{r}\right) \\
+\bar{q}_{D} \log \left(\bar{\mu}_{D}+v^{r}\right)+\underline{\bar{q}}_{D} \log \left(\underline{\bar{\mu}}_{D}+v^{r}\right)
\end{array}
$$

for $r<1$, and

$$
\begin{gathered}
\sum_{i} \frac{p_{i, \epsilon}}{1-\wp_{\epsilon}} \log \left(\frac{\Lambda_{i, \epsilon}}{D^{1 / r}}+v\right)^{r} \leq r\left[\underline{q}_{D} \log \left(\underline{\mu}_{D}^{1 / r}+v\right)\right. \\
\left.+\bar{q}_{D} \log \left(\bar{\mu}_{D}^{1 / r}+v\right)+\underline{\bar{q}}_{D} \log \left(\underline{\mu}_{D}^{1 / r}+v\right)\right]
\end{gathered}
$$

for $r \geq 1$. It follows that, for $r<1$, any sequence of quantizers satisfying (129) must also satisfy

$$
\begin{aligned}
\varliminf_{\kappa \downarrow 0} \varlimsup_{D \downarrow 0}\left\{\underline{q}_{D} \log \left(\underline{\mu}_{D}+v^{r}\right)+\bar{q}_{D} \log \left(\bar{\mu}_{D}+v^{r}\right)\right. \\
\left.+\underline{\underline{q}}_{D} \log \left(\underline{\bar{\mu}}_{D}+v^{r}\right)-\log \left(V+v^{r}\right)\right\} \geq 0 .
\end{aligned}
$$

Likewise, for $r \geq 1$, any sequence of quantizers satisfying (129) must also satisfy

$$
\begin{aligned}
& \underline{\lim }_{\kappa \downarrow 0} \varlimsup_{D \downarrow 0}\left\{\underline{q}_{D} \log \left(\underline{\mu}_{D}^{1 / r}+v\right)+\bar{q}_{D} \log \left(\bar{\mu}_{D}^{1 / r}+v\right)\right. \\
& \left.\quad+\underline{\bar{q}}_{D} \log \left(\underline{\mu}_{D}^{1 / r}+v\right)-\log \left(V^{1 / r}+v\right)\right\} \geq 0 .
\end{aligned}
$$

We conclude the proof of Theorem 7 for the case $r \geq 1$ by demonstrating that any sequence of quantizers satisfying (138b) must satisfy

$$
\lim _{\kappa \downarrow 0} \varlimsup_{D \downarrow 0} \bar{q}_{D}=1, \quad \text { for every } \vartheta>0 .
$$

Substituting $\rho=1 / \kappa$, this can be written as

$$
\lim _{\rho \rightarrow \infty} \varlimsup_{D \downarrow 0} \sum_{i} \frac{p_{i, \rho D^{1 / r}}}{1-\wp_{\epsilon}} \mathbf{1}\left\{\left|\frac{\Lambda_{i, \rho D^{1 / r}}^{r}}{D}-V_{1}^{r}(1+r)\right| \leq \vartheta\right\}=1
$$

for every $\vartheta>0$, which by (66)-(68) is equivalent to (78). The proof for $r<1$ is almost identical and is therefore omitted.

To prove (139) we first note that, by the strict concavity of $x \mapsto \log x$, there exists a linear function $x \mapsto \ell_{x_{0}}(x)$ such that

$$
\log (x+v) \leq \ell_{x_{0}}(x), \quad x \geq 0
$$

with equality if, and only if, $x=x_{0}$. (Specifically, we have $\ell_{x_{0}}(x)=\frac{x+v}{x_{0}+v}+\log \left(x_{0}+v\right)-1, x \geq 0$.) Moreover,

$$
\begin{aligned}
\log \left(\mu_{D}^{1 / r}+v\right)-\underline{q}_{D} \ell_{\mu_{D}^{1 / r}}\left(\underline{\mu}_{D}^{1 / r}\right) & \\
& -\bar{q}_{D} \ell_{\mu_{D}^{1 / r}}\left(\bar{\mu}_{D}^{1 / r}\right)-\underline{\bar{q}}_{D} \ell_{\mu_{D}^{1 / r}}\left(\underline{\mu}_{D}^{1 / r}\right) \geq 0
\end{aligned}
$$

since $x_{0} \mapsto \log \left(x_{0}+v\right)-\mathrm{E}\left[\ell_{x_{0}}(X)\right]$ (for any discrete random variable $X)$ is monotonically increasing and nonnegative for $x_{0} \geq \mathrm{E}[X]$, and since

$$
\underline{q}_{D} \underline{\mu}_{D}^{1 / r}+\bar{q}_{D} \bar{\mu}_{D}^{1 / r}+\underline{\bar{q}}_{D} \underline{\underline{\mu}}_{D}^{1 / r} \leq \mu_{D}^{1 / r} .
$$

The LHS of (138b) can thus be upper-bounded by

$$
\begin{aligned}
& \underline{q}_{D}\left[\log \left(\underline{\mu}_{D}^{1 / r}+v\right)-\ell_{\mu_{D}^{1 / r}}\left(\underline{\mu}_{D}^{1 / r}\right)-\log \left(\frac{V^{1 / r}+v}{\mu_{D}^{1 / r}+v}\right)\right] \\
& +\bar{q}_{D}\left[\log \left(\bar{\mu}_{D}^{1 / r}+v\right)-\ell_{\mu_{D}^{1 / r}}\left(\bar{\mu}_{D}^{1 / r}\right)-\log \left(\frac{V^{1 / r}+v}{\mu_{D}^{1 / r}+v}\right)\right] \\
& +\underline{q}_{D}\left[\log \left(\underline{\mu}_{D}^{1 / r}+v\right)-\ell_{\mu_{D}^{1 / r}}\left(\underline{\underline{\mu}}_{D}^{1 / r}\right)-\log \left(\frac{V^{1 / r}+v}{\mu_{D}^{1 / r}+v}\right)\right] .
\end{aligned}
$$

By (135) and (141), the third term in (143) satisfies

$$
\begin{aligned}
\varlimsup_{\kappa \downarrow 0} \varlimsup_{D \downarrow 0} \underline{\bar{q}}_{D}\left[\log \left(\underline{\mu}_{D}^{1 / r}+v\right)-\ell_{\mu_{D}^{1 / r}}\left(\bar{\mu}_{D}^{1 / r}\right)\right. \\
\left.-\log \left(\frac{V^{1 / r}+v}{\mu_{D}^{1 / r}+v}\right)\right] \leq 0 .
\end{aligned}
$$


We further have

$$
\begin{aligned}
\log & \left.\underline{\mu}_{D}^{1 / r}+v\right)-\ell_{\mu_{D}^{1 / r}}\left(\underline{\mu}_{D}^{1 / r}\right)-\log \left(\frac{V^{1 / r}+v}{\mu_{D}^{1 / r}+v}\right) \\
\leq & \log \left((V-\vartheta)^{1 / r}+v\right)-\ell_{(V+\varepsilon)^{1 / r}}\left((V-\vartheta)^{1 / r}\right) \\
& \quad+\log \left(\frac{(V+\varepsilon)^{1 / r}+v}{V^{1 / r}+v}\right) \\
\triangleq & \underline{\mathbf{K}}
\end{aligned}
$$

and

$$
\begin{aligned}
\log \left(\bar{\mu}_{D}^{1 / r}+v\right)-\ell_{\mu_{D}^{1 / r}}\left(\bar{\mu}_{D}^{1 / r}\right)-\log \left(\frac{V^{1 / r}+v}{\mu_{D}^{1 / r}+v}\right) \\
\leq \log \left((V+\vartheta)^{1 / r}+v\right)-\ell_{(V+\varepsilon)^{1 / r}}\left((V+\vartheta)^{1 / r}\right) \\
\quad+\log \left(\frac{(V+\varepsilon)^{1 / r}+v}{V^{1 / r}+v}\right) \\
\triangleq \overline{\mathrm{K}} .
\end{aligned}
$$

Here, we used (134) and (136) together with the facts that $x_{0} \mapsto \log \left(x_{0}+v\right)-\ell_{x_{0}}(x)$ is monotonically increasing, and that $x \mapsto \log (x+v)-\ell_{x_{0}}(x)$ is monotonically increasing for $x \leq x_{0}$ and monotonically decreasing for $x \geq x_{0}$.

Combining (143)-(146), it follows that (138b) can only be satisfied if

$$
\underline{\varliminf_{\kappa \downarrow}} \varlimsup_{D \downarrow 0} \max \{\underline{\mathrm{K}}, \overline{\mathrm{K}}\}\left(\underline{q}_{D}+\bar{q}_{D}\right) \geq 0 .
$$

Since $\max \{\underline{\mathrm{K}}, \overline{\mathrm{K}}\}$ does not depend on $D$ and, by (141),

$$
\lim _{\kappa \downarrow 0} \max \{\underline{K}, \bar{K}\}<0
$$

for $\varepsilon>0$ sufficiently small, the condition (147), in turn, can only be satisfied if

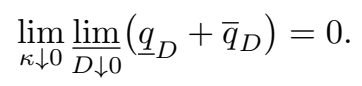

Using that $\underline{\bar{q}}_{D}=1-\underline{q}_{D}-\bar{q}_{D}$, the claim (139) follows. This concludes the proof of Theorem 7 .

\section{ACKNOWLEDGMENT}

Stimulating discussions with Tamás Linder and Ram Zamir are gratefully acknowledged. The authors further wish to thank Giuseppe Durisi for calling their attention to reference [17] and the Associate Editor David L. Neuhoff and the anonymous referees for their valuable comments.

\section{REFERENCES}

[1] C. E. Shannon, "A mathematical theory of communication," Bell System Techn. J., vol. 27, pp. 379-423 and 623-656, July and Oct. 1948.

[2] T. M. Cover and J. A. Thomas, Elements of Information Theory, 1st ed. New York, NY, USA: John Wiley \& Sons, 1991.

[3] A. D. Wyner, "An upper bound on the entropy series," Inform. Control, vol. 20, pp. 176-181, 1972.

[4] N. Alon and A. Orlitsky, "A lower bound on the expected length of oneto-one codes," IEEE Trans. Inf. Theory, vol. 40, no. 5, pp. 1670-1672, Sept. 1994.

[5] C. E. Shannon, "Coding theorems for a discrete source with a fidelity criterion," IRE International Convention Record, vol. 7, pp. 142-163, 1959.

[6] H. Gish and J. N. Pierce, "Asymptotically efficient quantizing," IEEE Trans. Inf. Theory, vol. 14, no. 5, pp. 676-683, Sept. 1968.
[7] R. M. Gray and D. L. Neuhoff, "Quantization," IEEE Trans. Inf. Theory, vol. 44, no. 6, pp. 2325-2383, Oct. 1998.

[8] Y. Yamada, S. Tazaki, and R. M. Gray, "Asymptotic performance of block quantizers with difference distortion measures," IEEE Trans. Inf. Theory, vol. 26, pp. 6-14, Jan. 1980.

[9] P. L. Zador, "Topics in the asymptotic quantization of continuous random variables," Bell Laboratories, Tech. Rep., 1966.

[10] Y. N. Linkov, "Evaluation of epsilon entropy of random variables for small epsilon," Problemy Peredachi Informatsii (Problems of Inform. Transm.), vol. 1, pp. 12-18, 1965.

[11] T. Linder and R. Zamir, "On the asymptotic tightness of the Shannon lower bound," IEEE Trans. Inf. Theory, vol. 40, no. 6, pp. 2026-2031, Nov. 1994.

[12] T. Koch, "The Shannon lower bound is asymptotically tight," IEEE Trans. Inf. Theory, vol. 62, no. 11, pp. 6155-6161, Nov. 2016.

[13] V. Kostina, "Data compression with low distortion and finite blocklength," in Proc. 53rd Allerton Conf. Comm., Contr. and Comp., Allerton H., Monticello, Il, Sep. 30 - Oct. 2, 2015.

[14] _ "Data compression with low distortion and finite blocklength," IEEE Trans. Inf. Theory, vol. 63, no. 7, pp. 4268-4285, July 2017.

[15] R. M. Gray, T. Linder, and J. Li, "A Langrangian formulation of Zador's entropy-constrained quantization theorem," IEEE Trans. Inf. Theory, vol. 28, no. 3, pp. 695-707, Mar. 2002.

[16] A. György and T. Linder, "On the structure of optimal entropyconstrained scalar quantizers," IEEE Trans. Inf. Theory, vol. 48, no. 2, pp. 416-427, Feb. 2002.

[17] Y. Wu and S. Verdú, "Rényi information dimension: Fundamental limits of almost lossless analog compression," IEEE Trans. Inf. Theory, vol. 56 , no. 8, pp. 3721-3748, Aug. 2010.

[18] A. Rényi, "On the dimension and entropy of probability distributions," Acta Mathematica Hungarica, vol. 10, no. 1-2, Mar. 1959.

[19] T. Kawabata and A. Dembo, "The rate-distortion dimension of sets and measures," IEEE Trans. Inf. Theory, vol. 40, no. 5, pp. 1564-1572, Sept. 1994.

[20] T. Linder and K. Zeger, "Asymptotic entropy-constrained performance of tessellating and universal randomized lattice quantization," IEEE Trans. Inf. Theory, vol. 40, no. 2, pp. 575-579, Mar. 1994.

[21] P. Billingsley, Probability and Measure, 3rd ed., ser. Wiley Series in Probability and Mathematical Statistics: Probability and Mathematical Statistics. New York, NY, USA: John Wiley \& Sons, 1995.

[22] M. S. Pinsker, Information and Information Stability of Random Variables and Processes, A. Feinstein, Ed. San Francisco: Holden-Day, 1964.

[23] S. Kullback and R. A. Leibler, "On information and sufficiency," Ann. Math. Statist., vol. 22, no. 1, pp. 79-86, 1951.

[24] R. B. Ash, Information Theory, ser. Dover Books on Mathematics. Mineola, NY, USA: Courier Dover Publications, 1990.

[25] A. Lapidoth and S. M. Moser, "Capacity bounds via duality with applications to multiple-antenna systems on flat fading channels," IEEE Trans. Inf. Theory, vol. 49, no. 10, pp. 2426-2467, Oct. 2003.

[26] F. Topsøe, "An information theoretical identity and a problem involving capacity," Studia Sci. Math. Hungar., vol. 2, pp. 291-292, 1967.

[27] I. Csiszár and J. Körner, Information Theory: Coding Theorems for Discrete Memoryless Systems, 2nd ed. Cambridge, UK: Cambridge University Press, 2011.

[28] T. Koch and A. Lapidoth, "On multipath fading channels at high SNR," IEEE Trans. Inf. Theory, vol. 56, no. 12, pp. 5945-5957, Dec. 2010.

[29] W. Yang, G. Durisi, and E. Riegler, "On the capacity of large-MIMO block-fading channels," IEEE J. Select. Areas Commun., vol. 31, no. 2, pp. 117-132, Feb. 2013.

[30] G. Villacrés and T. Koch, "Wireless networks of bounded capacity," in Proc. IEEE Int. Symposium on Inf. Theory, Barcelona, Spain, July 10-15, 2016.

[31] A. Lapidoth, "On phase noise channels at high SNR," in Proc. IEEE Inform. Theory Workshop (ITW), Bangalore, India, Oct. 20-25, 2002.

[32] M. Katz and S. Shamai (Shitz), "On the capacity-achieving distribution of the discrete-time noncoherent and partially coherent AWGN channels," IEEE Trans. Inf. Theory, vol. 50, no. 10, pp. 2257-2270, Oct. 2004.

[33] G. Durisi, A. Tarable, and T. Koch, "On the multiplexing gain of MIMO microwave backhaul links affected by phase noise," in Proc. ICC, Budapest, Hungary, June 9-13, 2013.

[34] A. Lapidoth and S. M. Moser, "On the capacity of the discrete-time Poisson channel," IEEE Trans. Inf. Theory, vol. 55, no. 1, pp. 303-322, Jan. 2009 
[35] A. Lapidoth, J. H. Shapiro, V. Venkatesan, and L. Wang, "The discretetime Poisson channel at low input powers," IEEE Trans. Inf. Theory, vol. 57, no. 6, pp. 3260-3272, June 2011.

[36] L. Wang and G. W. Wornell, "A refined analysis of the Poisson channel in the high-photon-efficiency regime," IEEE Trans. Inf. Theory, vol. 60, no. 7, pp. 4299-4311, July 2014.

[37] A. Lapidoth, S. M. Moser, and M. A. Wigger, "On the capacity of free-space optical intensity channels," IEEE Trans. Inf. Theory, vol. 55, no. 10, pp. 4449-4461, Oct. 2009.

[38] S. M. Moser, "Capacity results of an optical intensity channel with inputdependent Gaussian noise," IEEE Trans. Inf. Theory, vol. 58, no. 1, pp. 207-223, Jan. 2012

[39] H. Li, S. M. Moser, and D. Guo, "Capacity of the memoryless additive inverse Gaussian noise channel," IEEE J. Select. Areas Commun., vol. 32, no. 12, pp. 2315-2329, Dec. 2014.

[40] T. Koch, A. Lapidoth, and P. P. Sotiriadis, "Channels that heat up," IEEE Trans. Inf. Theory, vol. 55, no. 8, pp. 3594-3612, Aug. 2009.

[41] T. Koch, A. Martinez, and A. Guillén i Fàbregas, "The capacity loss of dense constellations," in Proc. IEEE Int. Symposium on Inf. Theory, Cambridge, MA, USA, July 1-6, 2012.

[42] J. H. Conway and N. J. A. Sloane, "A lower bound on the average error of vector quantizers," IEEE Trans. Inf. Theory, vol. 31, no. 1, pp. 106-109, Jan. 1985

[43] R. Zamir and M. Feder, "On lattice quantization noise," IEEE Trans. Inf. Theory, vol. 42, no. 4, pp. 1152-1159, July 1996

[44] R. B. Ash and C. A. Doléans-Dade, Probability and Measure Theory, 2nd ed. San Diego, CA, USA: Elsevier/Academic Press, 2000.

[45] E. M. Stein and G. Weiss, Introduction to Fourier Analysis on Euclidean Spaces. Princeton, NJ, USA: Princeton University Press, 1971.

Tobias Koch (S'02-M'09-SM'16) is a Visiting Professor and Ramón y Cajal Research Fellow with the Signal Theory and Communications Department of Universidad Carlos III de Madrid (UC3M). He received the M.Sc. degree in electrical engineering (with distinction) in 2004 and the Ph.D. degree in electrical engineering in 2009, both from ETH Zurich, Switzerland. From June 2010 until May 2012 he was a Marie Curie Intra-European Research Fellow with the University of Cambridge, UK. He was also a research intern at Bell Labs, Murray Hill, NJ, USA in 2004, and the Universitat Pompeu Fabra (UPF), Barcelona, Spain, in 2007. He joined the Signal Processing Group of UC3M in June 2012. His research interests are in digital communication theory and information theory.

Dr. Koch received a Starting Grant from the European Research Council (ERC), a Ramón y Cajal Research Fellowship, a Marie Curie Intra-European Fellowship, a Marie Curie Career Integration Grant, and a Fellowship for Prospective Researchers from the Swiss National Science Foundation. In 2013-2016 he served as Vice Chair of the Spain Chapter of the IEEE Information Theory Society.

Gonzalo Vazquez-Vilar (S'08-M'12) received the Telecommunication Engineering degree from the University of Vigo, Spain, in 2004, the Master of Science degree from Stanford University, U.S., in 2008 and the Ph.D. in Communication Systems from the University of Vigo, Spain, in 2011. Since 2014 he has been a Visiting Professor and Juan de la Cierva Fellow in the Signal Theory and Communications Department of Universidad Carlos III de Madrid, Spain. He has held research appointments at Stanford University, U.S., University of Cambridge, U.K., Universitat Pompeu Fabra, Spain, and Princeton University, U.S. His research interests lie in the field of Shannon theory, with emphasis in finite-length information theory. 